\title{
Egress of sperm autoantigen from seminiferous tubules maintains systemic tolerance
}

\author{
Kenneth S.K. Tung, ${ }^{1,2,3}$ Jessica Harakal, ${ }^{2,3}$ Hui Qiao, ${ }^{1,3}$ Claudia Rival, ${ }^{1,3}$ Jonathan C.H. Li, ${ }^{1}$ Alberta C.A. Paul, ${ }^{1}$ Karen Wheeler, ${ }^{1,3}$ \\ Patcharin Pramoonjago, ${ }^{1}$ Constance M. Grafer, ${ }^{1}$ Wei Sun, ${ }^{1}$ Robert D. Sampson, ${ }^{1,3}$ Elissa W.P. Wong, ${ }^{4}$ Prabhakara P. Reddi, \\ Umesh S. Deshmukh,, Daniel M. Hardy, ${ }^{6}$ Huanghui Tang, ${ }^{7}$ C. Yan Cheng, ${ }^{4}$ and Erwin Goldberg ${ }^{7}$ \\ 'Department of Pathology, ${ }^{2}$ Department of Microbiology, Immunology, and Cancer Biology, and ${ }^{3}$ Beirne B. Carter Center for Immunology Research, University of Virginia, Charlottesville, Virginia, USA. \\ ${ }^{4}$ Center for Biomedical Research, Population Council, New York, New York, USA. 'Department of Internal Medicine and Center of Regenerative Medicine and Immunity, University of Virginia, Charlottesville, \\ Virginia, USA. ${ }^{6}$ Cell Biology and Biochemistry, Texas Tech University Health Sciences Center, Lubbock, Texas, USA. Department of Molecular Biochemistry, Northwestern University, Evanston, Illinois, USA.
}

\begin{abstract}
Autoimmune responses to meiotic germ cell antigens (MCCA) that are expressed on sperm and testis occur in human infertility and after vasectomy. Many MGCA are also expressed as cancer/testis antigens (CTA) in human cancers, but the tolerance status of MCCA has not been investigated. MCCA are considered to be uniformly immunogenic and nontolerogenic, and the prevailing view posits that MCCA are sequestered behind the Sertoli cell barrier in seminiferous tubules. Here, we have shown that only some murine MCCA are sequestered. Nonsequestered MCCA (NS-MCCA) egressed from normal tubules, as evidenced by their ability to interact with systemically injected antibodies and form localized immune complexes outside the Sertoli cell barrier. NS-MGCA derived from cell fragments that were discarded by spermatids during spermiation. They egressed as cargo in residual bodies and maintained Treg-dependent physiological tolerance. In contrast, sequestered MGCA (S-MGCA) were undetectable in residual bodies and were nontolerogenic. Unlike postvasectomy autoantibodies, which have been shown to mainly target S-MCCA, autoantibodies produced by normal mice with transient Treg depletion that developed autoimmune orchitis exclusively targeted NS-MCCA. We conclude that spermiation, a physiological checkpoint in spermatogenesis, determines the egress and tolerogenicity of MGCA. Our findings will affect target antigen selection in testis and sperm autoimmunity and the immune responses to CTA in male cancer patients.
\end{abstract}

\section{Introduction}

Autoimmune responses to meiotic germ cell antigens (MGCA) expressed in the testis and on sperm are detected in $3 \%-12 \%$ of men with spontaneous infertility, which is a factor in $5 \%$ of couples desiring children (1). Testes biopsies have revealed that $50 \%$ of the patients had focal orchitis (2). Of the 0.5 million men in the USA who choose vasectomy as a contraceptive approach each year, 70\% develop sperm $\mathrm{Ab}$ responses with undefined long-term sequelae. In addition, MGCA are expressed as cancer/testis antigens (CTA) in many human epithelial tumors, melanomas, and leukemias. They are targets of tumor immunity and cancer vaccine candidates $(3,4)$. A better understanding of these tissue-specific and cancer-specific antigens in human diseases will require fuller knowledge of the sequestration and tolerance statuses of MGCA in normal and pathological states. Surprisingly, there have been little or no research efforts to address these critical issues.

Systemic tolerance is autoantigen dependent and involves multiple mechanisms (5). Tolerance begins in the thymus, controlled partly by the autoimmune regulator (AIRE) gene (6). This is followed by tolerance in the peripheral lymphoid

Authorship note: K.S.K. Tung and J. Harakal contributed equally to this work. Conflict of interest: The authors have declared that no conflict of interest exists. Submitted: August 4, 2016; Accepted: December 21, 2016.

Reference information: / Clin Invest. 2017;127(3):1046-1060.

https://doi.org/10.1172/JCl89927. organs, which requires continuous $\mathrm{T}$ cell recognition of accessible tissue autoantigens (7-9). Tregs are critical for preventing organ-specific autoimmunity (10-12). The maintenance of Treg function requires continuous $\mathrm{T}$ cell receptor (TCR) signaling (9) and autoantigen recognition (13) that occurs in the regional lymph nodes. Each regional lymph node expresses unique TCR clonotypes (14) and is enriched in tissue antigen-specific Tregs that preferentially suppress autoimmune disease of the draining organ $(8,15,16)$. Accordingly, peripheral tolerance should not apply to tissue autoantigens anatomically sequestered in organs such as the testes.

MGCA are expressed in postmeiotic germ cells located in the seminiferous tubules (Figure 1A), separated from interstitial leukocytes by the basal tight junctions of the Sertoli cell barrier (Figure 1, B and C) (17). Rodent MGCA appear 2 weeks after birth (Figure 1, B and C) (18); thereafter, a huge quantity of numerous novel testis-specific antigens are continuously synthesized and expressed in the meiotic germ cells (19). After being released into the seminiferous tubule lumen, the sperm enter the rete testis and ultimately reach the vas deferens (Figure 1A) (19). Spermatogenesis in adult mice occurs in 12 distinct stages along the length of the seminiferous tubule, each defined by unique composition and arrangement of the germ cells (Figure 1A) (20). Because the stages differ among tubules, their cross sections in an adult testis in many different stages coexist. However, the first wave of spermatogenesis initiated at puberty is synchronous in all tubules (21). 
A
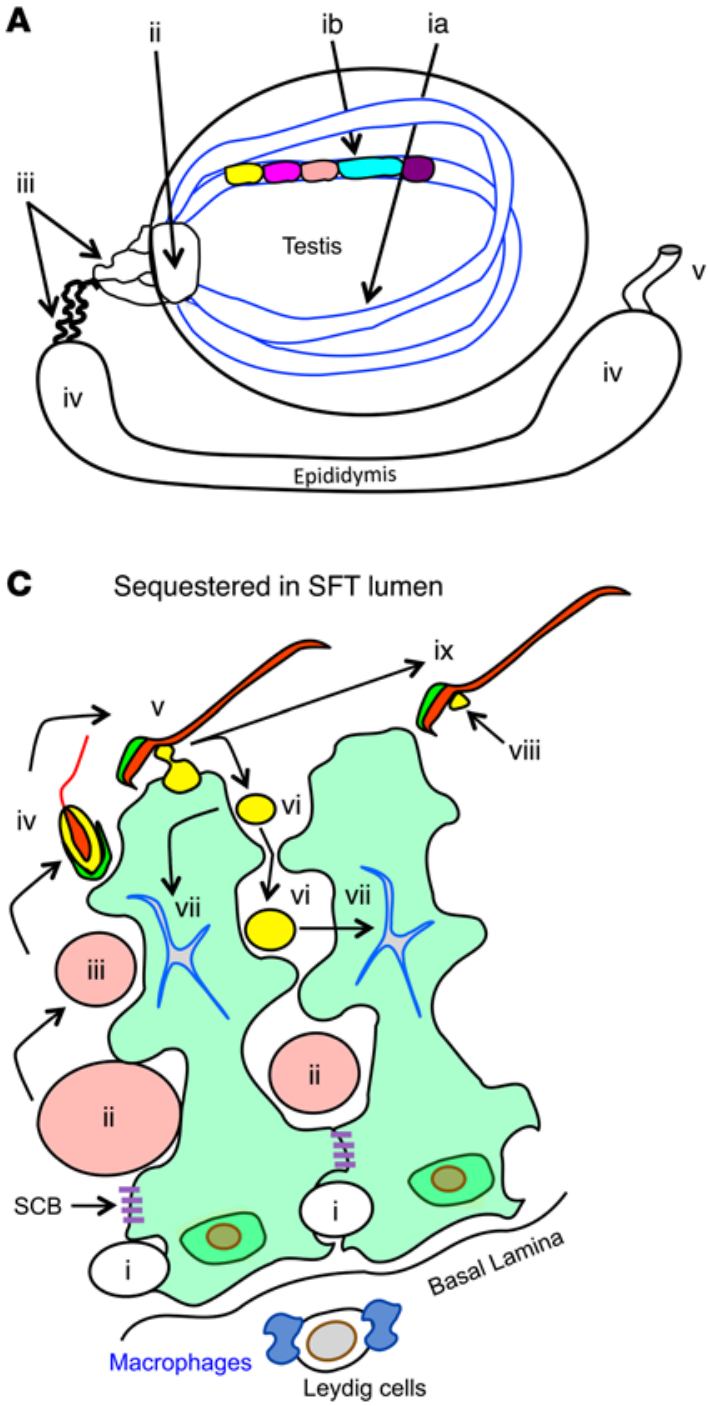

B
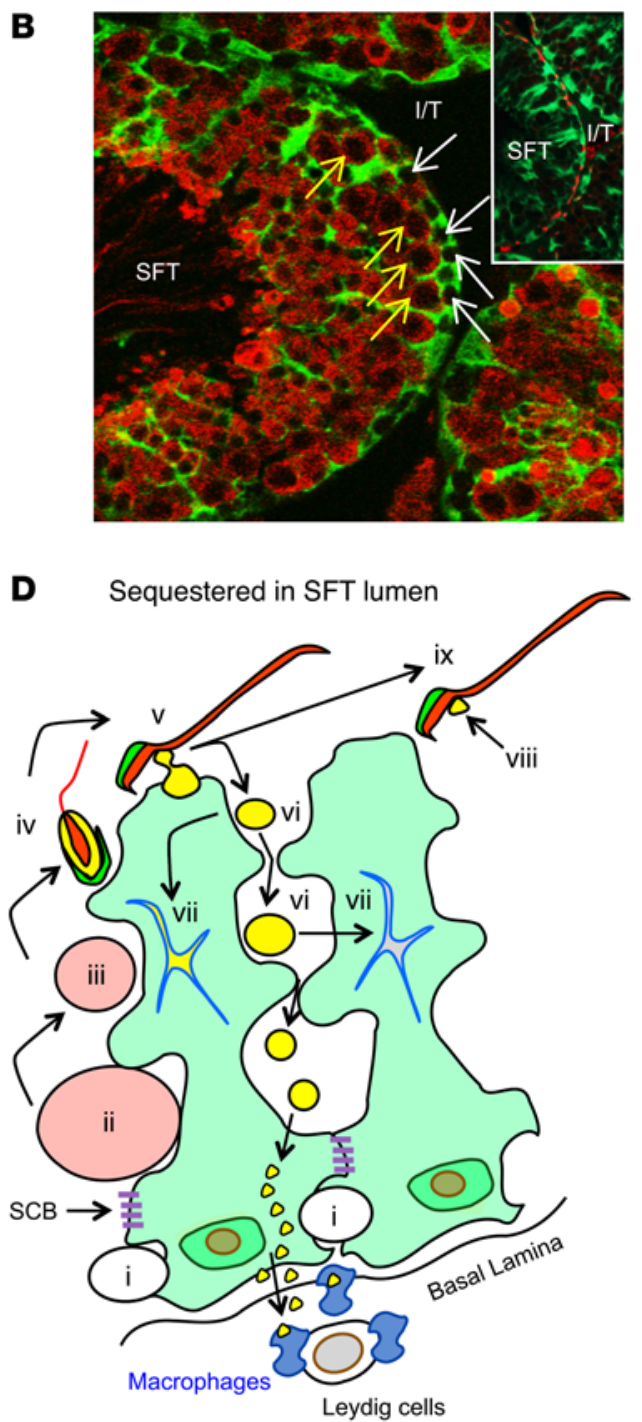

Figure 1. Testis anatomy and selective MGCA sequestration. (A) Sperm production occurs in cycles (different colors) longitudinally along seminiferous tubules (SFT) (ia, ib). Mature sperm are transported to the vas deferens (v) via the rete testis (ii), ductus efferentes (iii), and epididymis (iv). (B) Seminiferous tubules, visualized by IF on Scx-GFP mouse testes with GFP+ Sertoli cells (green) and LDH3 (red), contain LDH3- spermatogonia (white arrows) and $\mathrm{LDH}^{+}$spermatocytes (yellow arrows). Insert: occludin+ Sertoli cell barriers (red) located between adjacent Sertoli cells (green). I/T, interstitial space. Original magnification, $\times 500 ; \times 800$ (insert). (C) A seminiferous tubule segment with 2 Sertoli cells (light green) depicts the complete MGCA sequestration paradigm. Sertoli cells support spermatogenesis (steps i-iv) and spermiation (steps v-ix). Spermatogonia (i) traverse the Sertoli cell barriers to become MGCA+ spermatocytes (ii, pink), then round (iii) and elongating (iv) spermatids. At spermiation, redundant cytoplasm (yellow) and plasma membrane (black) are partially detached from elongated spermatids (v) to form residual bodies (vi) destined for degradation inside Sertoli cells (vii), and retained as cytoplasmic droplets (viii) on mature sperm (ix). The interstitial space contains spermatogonia, basal lamina, peritubular cells (not shown), Leydig cells, macrophages, and afferent lymphatic vessels (not shown). Sertoli cells and Sertoli cell barriers (purple) sequester all MGCA meiotic germ cells inside seminiferous tubules. (D) The new selective MGCA sequestration paradigm supported by our study is shown. Tolerogenic NS-MGCA are located in residual bodies and not removed by Sertoli cells. They enter the basal Sertoli cell cytoplasm and egress into the interstitial space. S-MCCA, including those in the sperm acrosome (green crescent), absent from residual bodies, are nontolerogenic. Note that some, but not all, residual bodies are destroyed by the Sertoli cells (vii).

Because the Sertoli cell barrier effectively blocks Abs and lymphoid cells from entering the seminiferous tubules (22-24), the barrier is presumed to also prevent MGCA from entering the interstitial space to reach the immune cells (19, 25-27). Accordingly, local antigen nonspecific mechanisms are deemed sufficient to control tissue barrier integrity and excessive testis inflammation in autoimmunity, trauma, or infection $(19,25,27)$. However, the complete antigen sequestration paradigm itself has neither been investigated nor supported by experimental evidence, and importantly, its validity has been questioned by recent clinical and experimental findings.

First, consistent with other spontaneous autoimmune diseases, the cause of autoimmune infertility is likely rooted in immune dysregulation or defective tolerance and is influenced by genetic and environmental components. Therefore, loss of tolerance rather than loss of sequestration is the likely cause of autoimmune 

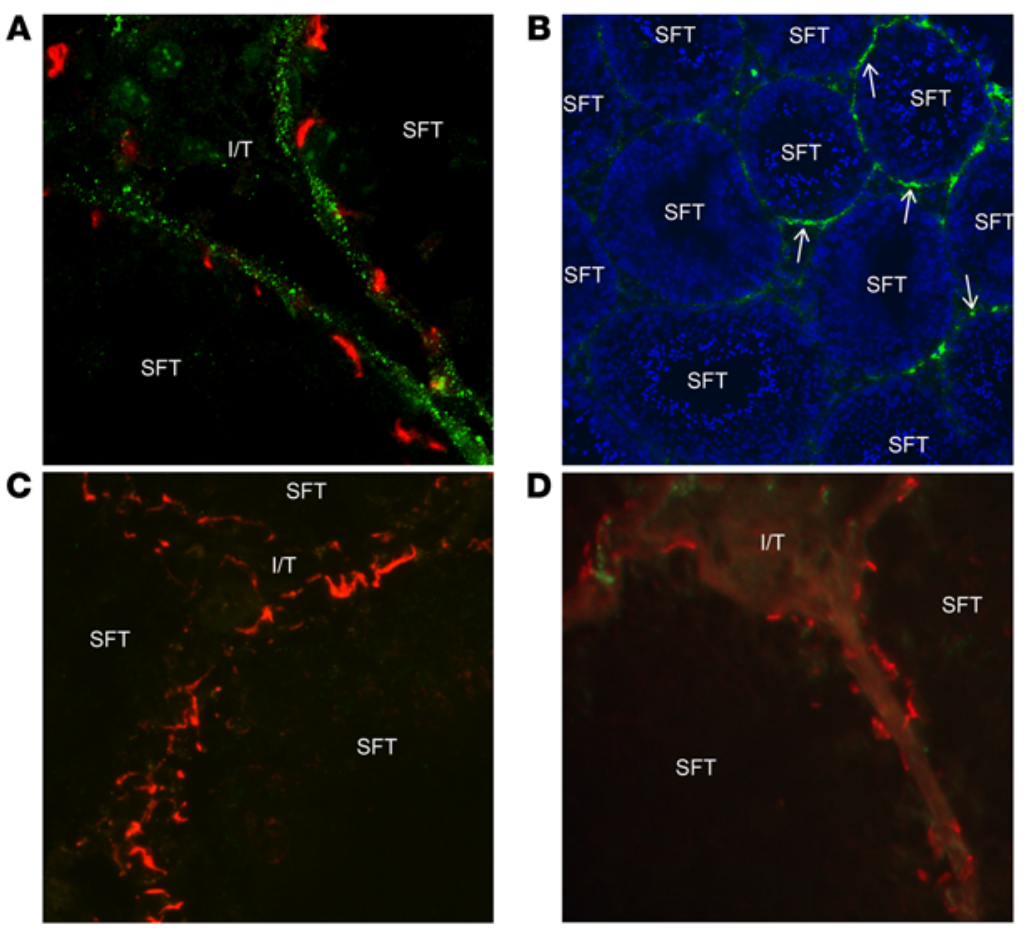

Figure 2. Tolerogenic MGCA egress from seminiferous tubules of normal mouse testes. (A) After rabbit anti-LDH3 Ab injection, immune complexes appeared as rabbit IgC puncta (green) outside the occludin ${ }^{+}$Sertoli cell barrier (red) in adult WT mouse testes $(n=7)$ surrounding approximately $18 \%$ of seminiferous tubules (B arrows, $n=5$ ), but not in (C) testes of adult $L d h 3$ null mice $(n=7)$. (D) No immune complexes were detected in the testes of WT mice injected with rabbit anti-ZAN D3p18 Abs. Original magnification, $\times 800$ (A); $\times 100$ (B); $\times 800$ (C); $\times 800$ (D). Tolerance to LDH3 and ZAN were determined by serum IgC Ab responses to rLDH3 (E and F) or ZAN D3p18 (G) at 3 weeks after immunization with testis homogenate in adjuvant. (E) Comparison of rLDH3 responses between WT male mice $(n=5)$ and WT female mice $(n=5)$ and $(\mathbf{F})$ between $L d h 3$ null male mice $(n=6)$ and WT male mice $(n=6)$ are shown. (C) Comparison of ZAN D3p18 responses between WT male mice $(n=5)$ and WT female mice $(n=3)$ is shown. Ab responses to $(\mathbf{H})$ rLDH3 and (I) ZAN in WT male mice at 3 weeks after immunization with testis homogenate in adjuvants, with $(n=7)$ and without ( $n=13$ ) concomitant CD25 mAb (PC61) injection are shown. Data in E-I are from 3 independent experiments. ${ }^{*} P<0.05$; ${ }^{* *} P<0.01$, Mann-Whitney $U$ tests.
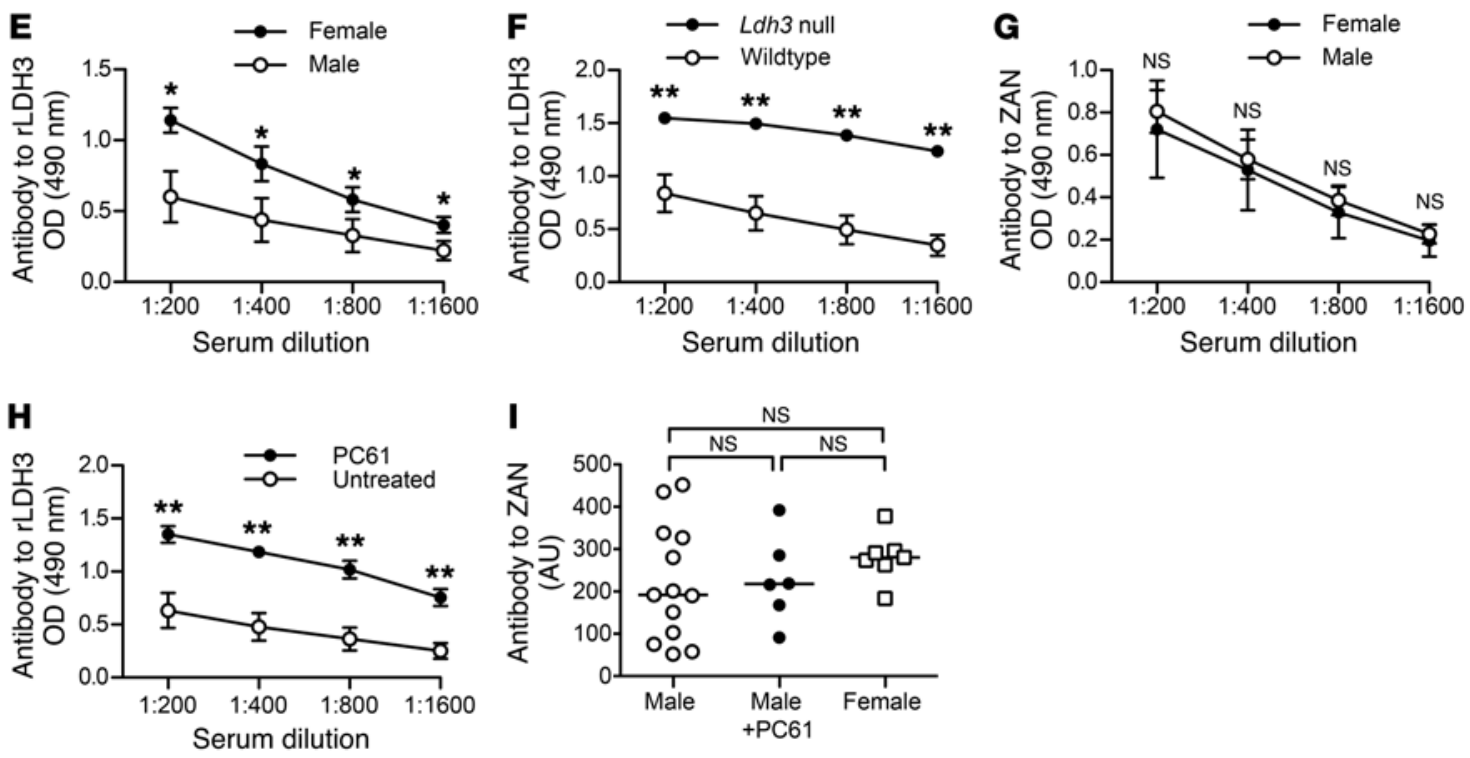

infertility. For example, autoimmune orchitis occurs in autoimmune polyendocrine syndrome 1 (APS1) patients due to mutations of AIRE (28), possibly associated with impaired thymic deletion of autoreactive T cells and deficient Treg function (29).

Second, vasectomy, a popular contraceptive approach (30), does cause leakage of sperm in the epididymis (31). Instead of the expected MGCA Ab response directed to the numerous sequestered MGCA, a recent study indicated that over $85 \%$ of the vasectomized mice produced a dominant autoantibody response against zonadhesin (ZAN) (32), an MGCA located in the outer acrosomal membrane of spermatids and the acrosomal matrix of spermatozoa $(33,34)$. Moreover, an unexplained latent period of several months exists before vasectomy results in detectable MGCA autoantibody responses (35). In this period, vasectomized mice have recently been found to develop a prolonged period of MGCA-specific tolerance due to the emergence of MGCA-specific Tregs (36). Thus, postvasectomy autoimmunity occurred only when it was accompanied by partial Treg depletion with an anti-CD25 mAb $(32,36)$, a finding more likened to the concomitant effector $\mathrm{T}$ cell and Treg responses to foreign antigens described in food allergy (37) and in viral infections (38). Therefore, postvasectomy responses resemble the responses to immunogenic "foreign" antigens; moreover, the $\mathrm{Ab}$ repertoire is narrower than expected and suggests the existence of tolerance to other MGCA (36).

Third, a recent study on lactate dehydrogenase 3 (LDH3) (the mouse homolog of human LDHC) also argues against complete MGCA sequestration. LDH3 is a cytoplasmic MGCA expressed behind the Sertoli cell barrier and is a CTA $(18,39)$. After systemic 
injection, liposomes bearing LDH3 Abs were enriched in the testes of normal mice, indicating that LDH3 may be accessible to circulating Abs in vivo (40).

In this study, we address whether all MGCA are sequestered and whether they are protected by systemic tolerance. Our study focuses on LDH3 and ZAN in WT mice. We also generated 2 transgenic mouse lines that express OVA in elongated spermatids, allowing us to investigate and identify the route of MGCA egress. We also developed a new model of spontaneous experimental autoimmune orchitis (EAO) to explore defective systemic tolerance as a mechanism of autoimmune infertility. We showed that some MGCA are not sequestered. They exit the seminiferous tubules via a previously unappreciated physiological pathway that is important for testis function and maintain systemic tolerance. Finally, we documented that systemic tolerance to nonsequestered MCGA is critically dependent on Tregs. Conversely, other MGCA are sequestered and not tolerogenic.

\section{Results}

Tolerogenic MGCA continuously egress from the seminiferous tubules of normal mouse testes. To investigate the sequestration status of LDH3 and ZAN, we localized the tissue distribution of their cognate Abs in WT testes after systemic injection. The Sertoli cell barrier is not an endothelial barrier, and circulating Abs readily enter the testicular interstitial space (19). However, being excluded from the seminiferous tubules by the Sertoli cell barrier, the Abs would react with NS-MGCA, but not with sequestered MGCA (S-MGCA), outside the barrier.

Patches of granular rabbit anti-LDH3 IgG were observed at the boundaries of the seminiferous tubules 19 hours after Ab injection (Figure 2A). The extratubular location of the immune complexes was confirmed by costaining with occludin, a Sertoli cell barrier component. This pattern was reproducible in $83 \%$ of 35 mice on different mouse strains, including C57BL/6 $(n=10), \mathrm{BALB} / \mathrm{c}$ $(n=10)$, SJL $(n=4)$, and C57BL/6 $\times$ A/J F1 (B6AF1; $n=11)$. Because immune complexes were only detected in approximately $18 \%$ of seminiferous tubule cross sections (Figure $2 \mathrm{~B}, n=6$ ), we speculated that the observed LDH3 egress may occur only at some stages of spermatogenesis (20), a possibility that we address later in this study. Importantly, immune complexes were not detected in the testes of $L d h 3$ null C57BL/6 mice (41) (Figure 2C, $n=7$ ), but were detected in $L d h 3^{+/-}$mice $(n=5)$. They were also not detected in mice injected with rabbit IgG or rabbit anti-OVA Abs $(n=13)$. In contrast with mice injected with $\mathrm{LDH} 3$, mice injected with Abs to ZAN at several dilutions did not show testicular immune complexes. This applies to 2 ZAN Abs, one against the D3p18 B cell epitope of mouse ZAN (Figure 2D) and the other against the holoprotein of pig ZAN that crossreacts with mouse ZAN (data not shown). Therefore, although both MGCAs are located behind the Sertoli cell barrier, LDH3 selectively egresses from the seminiferous tubules, whereas ZAN is sequestered.

To define the tolerance status for LDH3 and ZAN, we compared the LDH3- and ZAN-specific responses of male and female mice after immunization with testis homogenate in adjuvant. A stronger $\mathrm{Ab}$ response to $\mathrm{LDH} 3$ was detected in female mice compared with male mice (Figure 2E). Importantly, the response of Ldh3 null male mice with normal spermatogenesis also surpassed that of WT male mice (Figure $2 \mathrm{~F}$ ). In contrast to LDH3, ZAN Ab responses were comparable between the immunized male and female mice (Figure $2 \mathrm{G}$ ). These results, together with the findings on cognate $\mathrm{Ab}$ localization, suggest that $\mathrm{LDH} 3$ is a tolerogenic NS-MGCA, whereas ZAN is a nontolerogenic S-MGCA.

$\mathrm{CD}^{+} \mathrm{CD} 25^{+} \mathrm{FOXP} 3^{+}$Tregs control peripheral tolerance to the NS-MGCA. We began by treating the testis homogenate-immunized male WT B6AF1 mice with anti-CD25 mAb that depletes approximately $60 \%$ of Tregs for 5 weeks (32). This significantly enhanced the $\mathrm{LDH} 3 \mathrm{Ab}$ responses compared with untreated male mice (Figure $2 \mathrm{H}$ ), but did not affect ZAN Ab responses (Figure 2I). Therefore, Tregs are critical in controlling systemic tolerance of WT mice to LDH3.

To accrue more definitive evidence for Treg involvement in physiological tolerance to MGCA, we conducted experiments independent of testis antigen immunization in BAC-transgenic DEREG mice that express a diphtheria toxin (DT) receptor under the control of the forkhead box P3 (Foxp3) locus (42). Treg depletion by DT injections in B6AF1-DEREG mice resulted in the production of serum autoantibodies against testicular cell antigens. The response began at 3 weeks (Figure 3A) and was detected in $72 \%$ of the mice at 8 weeks (Figure $3 \mathrm{~B}$ ). Notably, the autoantibodies reacted only with the tolerogenic and nonsequestered LDH3 (Figure 3C) and not with the sequestered ZAN (Figure 3D).

In addition to autoantibody responses, severe EAO was detected in $40 \%$ of the Treg-depleted DEREG mice at 8 weeks (Figure 3E) and could be suppressed by adoptive transfer of WT Tregs. EAO was characterized by disruption of spermatogenesis (Figure 3, E and F), reduction in epididymal sperm (Figure 3, E and F), focal interstitial lymphocytic infiltration (Figure 3F), reduction in testis weight (Figure 4A), and tubular atrophy with abundant interstitial macrophage infiltration (Figure $3 \mathrm{~F}$ and Figure $4 \mathrm{~B}$ ). A larger fraction and number of the macrophages exhibited a proinflammatory EAO-associated M1-like phenotype as distinct from the M2-like phenotype of normal testis-resident macrophages $(43,44)$ (Figure 4 C). The mice with epididymi devoid of sperm are expected to be infertile.

Although only $40 \%$ of the Treg-depleted DEREG mice exhibited testis histopathology, many more $(72 \% ; n=44)$ developed massive immune complex deposition. Diffusely distributed immune complexes were detected at the seminiferous tubule boundaries adjacent to the defective Sertoli cell barrier (Figure 4D). They were mostly of IgG1 subclass without IgG2a or C3 (data not shown). In addition, defective Sertoli cell barrier integrity was documented by the detection of subcapsularly injected biotin inside the seminiferous tubule lumen (Figure 4E). The global increase in Sertoli cell barrier protein expression (Figure 4F) was indicative of the Sertoli cells' adaptive responses in restoring tight junction integrity (45). Moreover, i.v.-injected rabbit LDH3 Ab into DEREG mice with EAO formed rabbit IgG-positive immune complexes that comingled with the endogenous mouse IgG1-positive immune complexes inside the seminiferous tubules (Figure $4 G)$. Notably, a reduction of nectin-3, which anchors elongated spermatids to the Sertoli cells, was consistent with elongated spermatid loss (Figure 4F). The results indicate that Tregs maintain physiological tolerance to the NS-MGCA that egress from the seminiferous tubules, but not to the S-MGCA. 
A

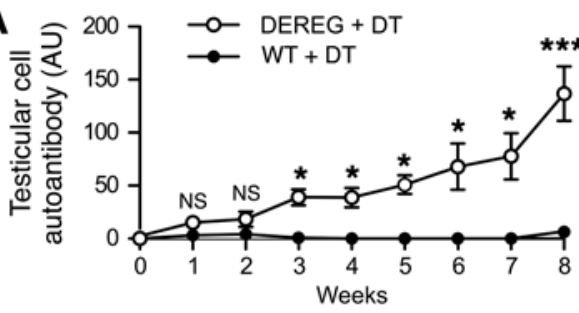

B

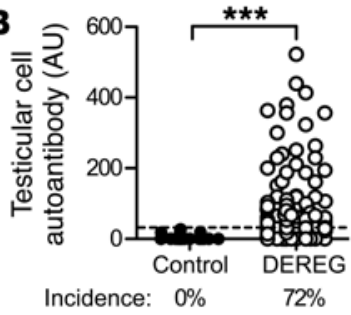

C

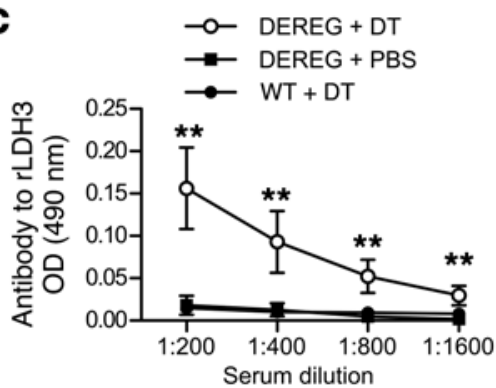

D

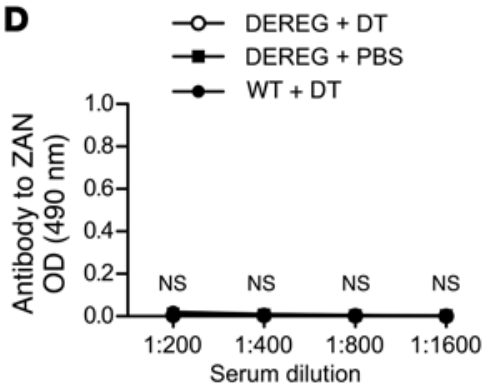

Figure 3. Treg-depleted B6AF1-DEREC mice spontaneously develop autoimmune orchitis and produce Abs to LDH3 but not to ZAN. (A) Kinetics of autoantibody responses to testicular cell antigens $(n=3-43$ per time point). (B) Incidence and level of testicular cell autoantibody at 8 weeks between Treg-depleted DEREG mice $(n=106)$ and WT mice $(n=22)$ treated with DT. Serum Ab to (C) rLDH3 ( $n=5$ to $n=10$ per group) and (D) ZAN D3p18 ( $n=5$ to $n=10$ per group) in control and Treg-depleted DEREG mice at 8 weeks. (E) Incidences and severity of seminiferous tubules with abnormal spermatogenesis and sperm loss in epididymis at 8 weeks. (F) Representative histopathology of testis and epididymis between DEREC mice treated with DT (bottom) and PBS (top). Arrow points to a cluster of leukocyte-like cells in the tubule lumen $(H \& E)$. Original magnification, $\times 400$ (testis); $\times 200$ (epidymis). Data are pooled from 6-12 independent experiments. ${ }^{*} P<0.05$; ${ }^{* *} P<0.01$; ${ }^{* *} P<0.001$, Mann-Whitney $U$ tests (A-D); Kruskal-Wallis tests with Dunn's post-tests (E).
$\mathbf{E}$

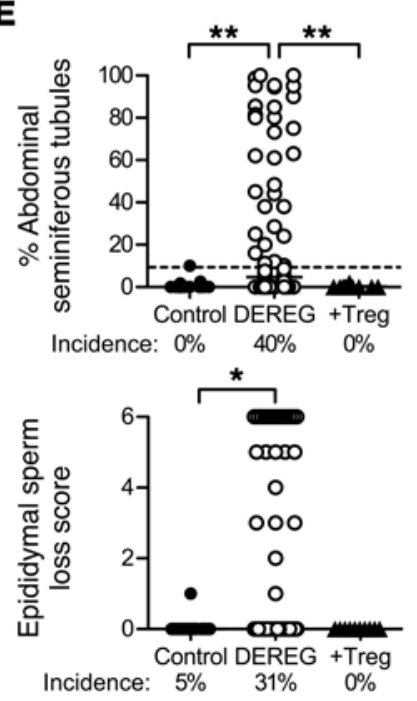

$\mathbf{F}$
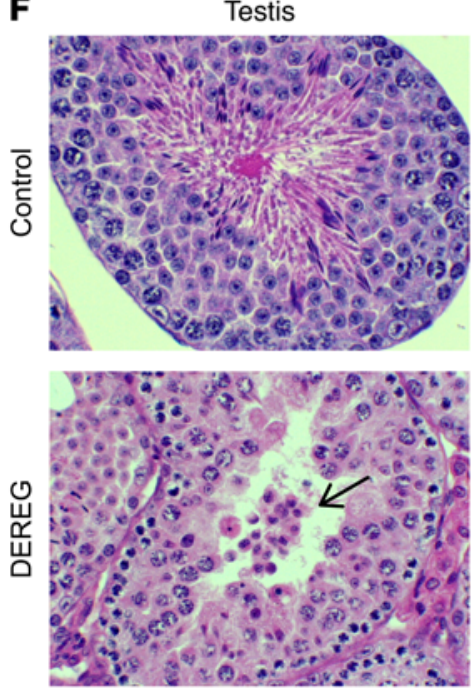

Epididymis
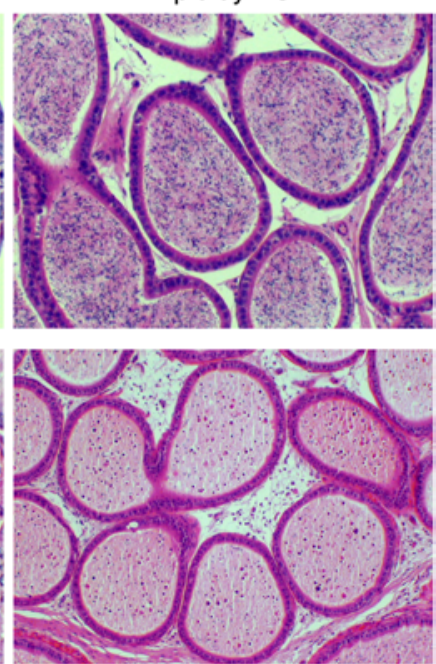

OVA expressed as a surrogate MGCA in elongated spermatids also egresses from the seminiferous tubules and depends on the level of expression. We next extended our study to 2 founder lines of OVA transgenic mice on the BALB/cByJ background, in which OVA was expressed as a surrogate MGCA under the mouse protamine 1 (Prm1) promoter to ensure testis-specific OVA expression (46) (Figure 5A). OVA-Hi mice expressed $2200 \pm 45 \mathrm{ng}$ of extractable OVA/g of testis, and OVA-Lo mice expressed 41-fold less OVA, at $54 \pm 15 \mathrm{ng} / \mathrm{g}$ of testis. Both OVA-Hi and OVA-Lo mice expressed OVA confined to the elongated spermatid cytoplasm (Figure 5B). They exhibited normal fertility (data not shown) and normal testis histology (Figure 5B) and epididymides with full sperm content (data not shown). Consistent with elongated spermatid development (20), extractable OVA protein and OVA mRNA levels from OVA-Hi mouse testes were initially detected at postnatal day 25 and markedly increased in the next 4 days (Figure 5C and data not shown). Moreover, the Sertoli cell barrier was intact, as determined by electron microscopy (Figure 5D), and the seminiferous tubules excluded the FITC injected into the interstitial space (Figure 5E).
OVA also egressed from the seminiferous tubules in mice expressing high levels of OVA. Deposition of granular rabbit antiOVA IgG immune complexes was readily detected in approximately $18 \%$ of 214 seminiferous tubules in $100 \%$ of OVA-Hi mice after i.p. injection of rabbit anti-OVA Abs $(n=13$, Figure 5F), but not control rabbit IgG ( $n=7$, data not shown). These immune complexes were located outside the Sertoli cell barrier. Some immune complexes were also detected inside the testis interstitial MHC class $\mathrm{II}^{+}$macrophages (Figure $5 \mathrm{G}$ ). In contrast, only trace immune complexes were detected in less than $5 \%$ of seminiferous tubules in 4 of 5 OVA-Lo mice injected with rabbit anti-OVA Abs.

Our results indicate that (a) the testicular structural and functional properties of the OVA-Hi and OVA-Lo mice are indistinguishable from those of WT BALB/c mice, (b) OVA also egresses from the OVA-Hi mouse testes similarly to LDH3 in WT mice, and (c) OVA egress is dependent on the level of OVA expression behind the Sertoli cell barrier. We therefore utilized both the transgenic and WT mice to investigate the mechanism of MGCA egress.

Residual bodies export NS-MGCA, but not S-MGCA. We next investigated how NS-MGCA egress from normal seminiferous 
A

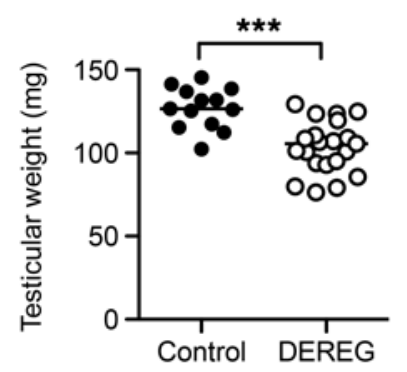

B

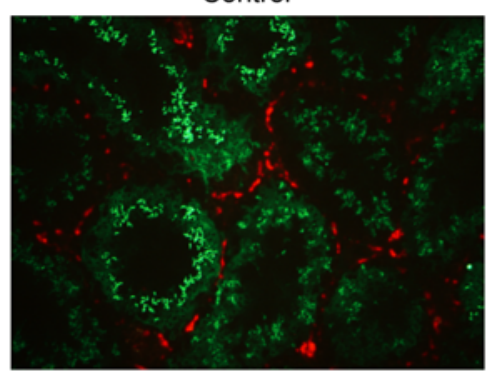

DEREG

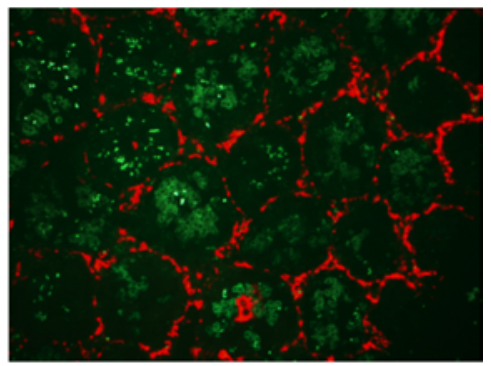

C
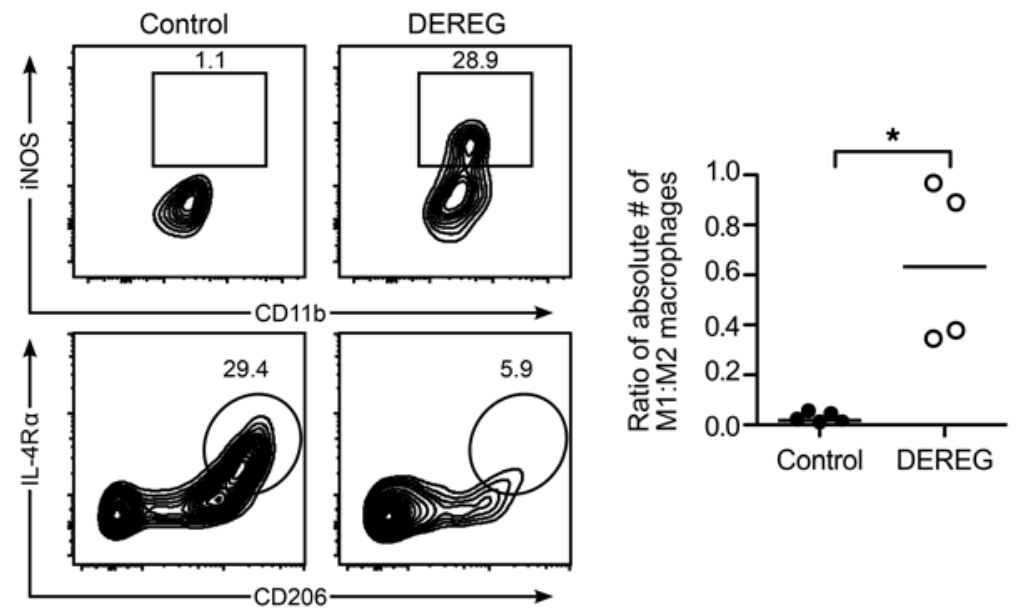

D

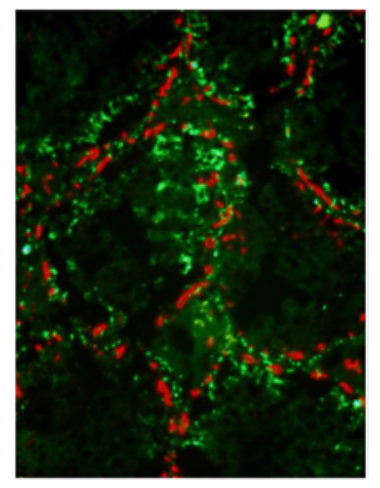

G

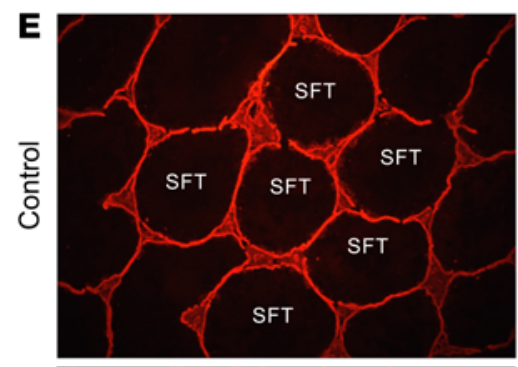

F Control DEREG

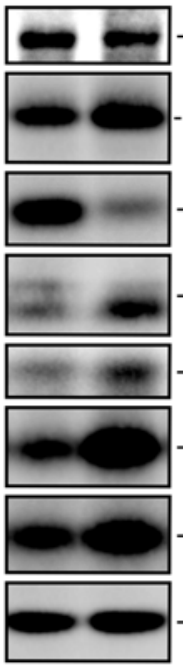

ZO-1, $210 \mathrm{kDa}$

$\beta$-Catenin, $92 \mathrm{kDa}$

- Nectin-3, $83 \mathrm{kDa}$

- Occludin, $65 \mathrm{kDa}$

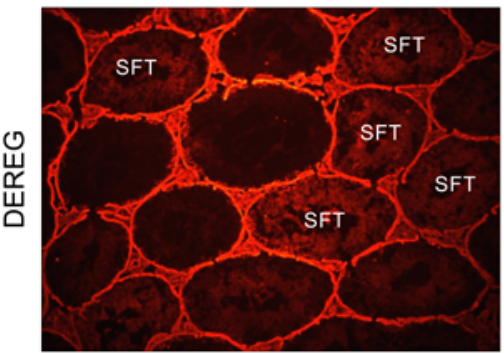

- JAM-C, $45 \mathrm{kDa}$

- Connexin 43, $39 \mathrm{kDa}$

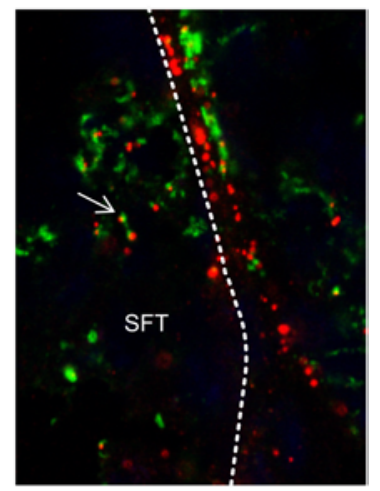

- JAM-A, $36 \mathrm{kDa}$

- Actin, 42 kDa

Figure 4. EAO of Treg-depleted DEREG mice is characterized by M1-like macrophage infiltration, immune complex deposition, and disrupted Sertoli cell barrier. (A) Testis weight at 8 weeks after DT treatment. (B) Increased interstitial F4/80+ macrophages (red) and reduced seminiferous tubule diameter and ZAN ${ }^{+}$spermatids (green) in DEREG mice with EAO compared with control mice. Original magnification, $\times 100$. (C) Most macrophages of control mice have the characteristic phenotype of M2 macrophages, including the expression of IL-4R $\alpha$, CD206, and F4/80 (left), whereas macrophages of Treg-depleted DEREG mice have an M1-like phenotype of high iNOS and MHC class II expression (right); data are expressed as a ratio of absolute numbers (right), where each symbol represents cells from 2 testes of 1 mouse. (D) Mouse IgG immune complexes (green) in the DEREG mouse testes with EAO are located both outside and inside seminiferous tubules relative to the occludin ${ }^{+}$Sertoli cell barrier (red). Original magnification, $\times 300$. (E) Biotin (red) exclusion assay in testes of control mice (top) and Treg-depleted DEREG mice (bottom). Original magnification, $\times 100$. (F) Western blot analysis of extractable tight junction proteins of the Sertoli cell barrier from testes of control and Treg-depleted DEREC mice. This is a composite data set of 8 parallel blots wherein 4 control and Treg-depleted DEREG mouse testis samples were analyzed by using corresponding specific Abs in a single experimental session to avoid interexperimental variations. (G) IF staining of murine IgG (green) and rabbit IgG (red) in the testis of a DEREG mouse with EAO at 19 hours after i.p. injection of rabbit Abs to LDH3. Note rabbit (red) and mouse (green) immune complexes were admixed or colocalized inside the seminiferous tubule (arrow). Dotted line, seminiferous tubule boundary. Original magnification, $\times 800$. Data are from 2-12 independent experiments. ${ }^{*} P<0.05 ;{ }^{* *} P<0.001$, Mann-Whitney $U$ test $(\mathbf{A}$ and $\mathbf{C})$. 
A

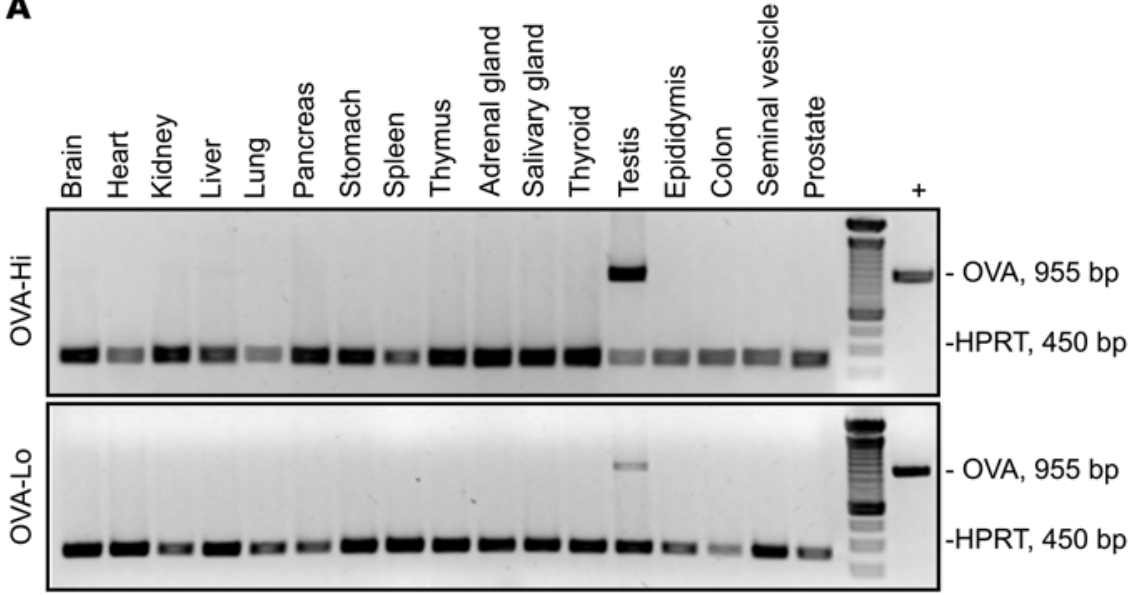

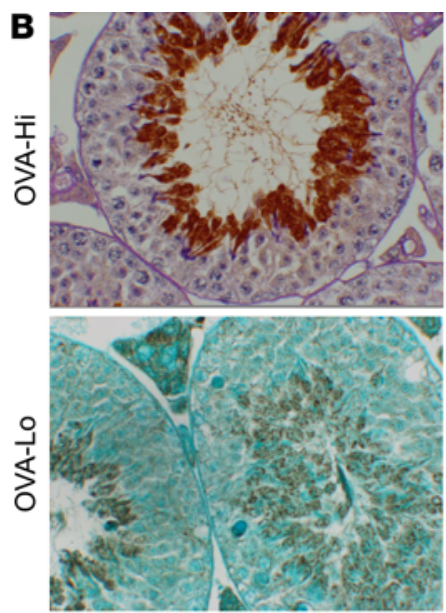
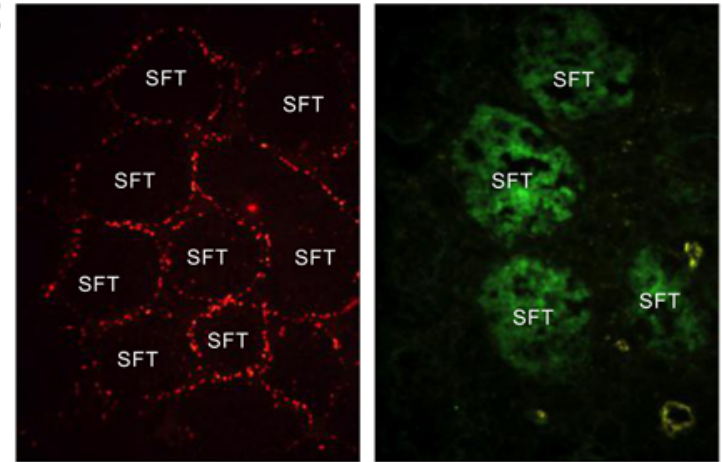

$\mathbf{F}$

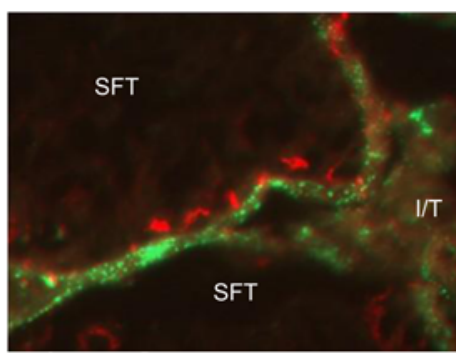

D

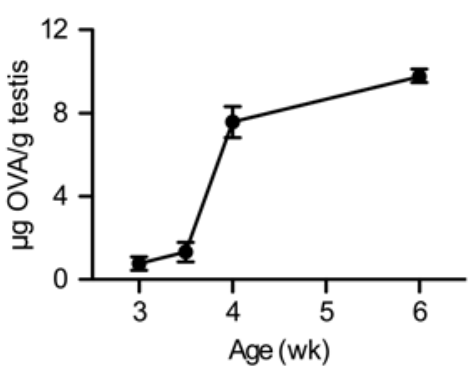

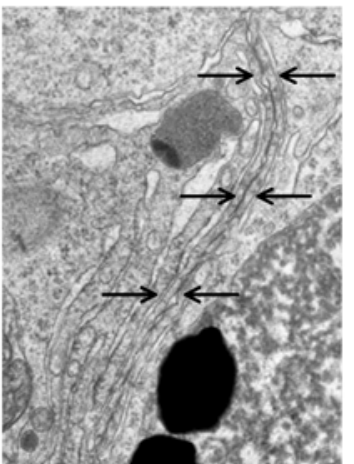

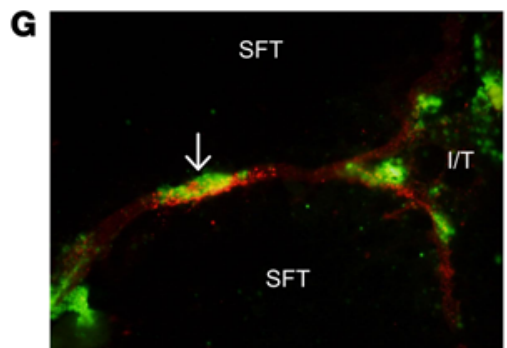

Figure 5. Testis-specific transgenic OVA expressed in elongated spermatids as a surrogate MGCA also egresses from seminiferous tubules in a concentration-dependent manner. Comparison between OVA-Hi and OVA-Lo mice: (A) adult OVA expression (reverse transcriptase PCR [RT-PCR]) and (B) OVA localization in elongated spermatids (top, IP with PAS; bottom, IP with hematoxylin). (C) Ontogeny of testicular OVA expression in OVA-Hi mice (ELISA). (D) Tight junction ultrastructure of a Sertoli cell barrier (arrows) in adult OVA-Hi mouse testis. (E) FITC (green) exclusion by the occludin ${ }^{+}$Sertoli cell barrier (red) of seminiferous tubules in an OVA-Hi mouse testis (left) compared with testis of a mouse with EAO (right). In OVA-Hi mice injected i.p. with rabbit Abs to OVA, (F) immune complexes detectable as rabbit IgG puncta (green) are detected outside the occludin+ Sertoli cell barrier (red) and (G) inside interstitial MHC class $\mathrm{II}^{+}$macrophages (green, arrow). Original magnification, $\times 400$ (B); $\times 10,000$ (D); $\times 800$ (E); $\times 400$ (F-C).

tubules and whether this is dependent on spermatogenic stages. Because the cycles in all seminiferous tubules are synchronous in the first wave of spermatogenesis, it is possible to define the age of onset of immune complex deposition and accurately define the stage of the cycle when MGCA egress occurs. LDH3 Ab injection at 5 weeks, but not at 3,4 , or 4.5 weeks, after birth, elicited immune complexes in many tubules ( $n=4$ for each group) (Figure $2 \mathrm{~B}$ and data not shown). This timing is consistent with the timing of spermiation when the mature testicular sperm are released into the seminiferous tubule lumen (21), but not with LDH3 expression that begins at postnatal day 12 (18).
Prior to sperm release, the redundant cytoplasm and plasma membrane of elongated spermatids are packaged into a large cytoplast (the residual body) retained in the seminiferous tubule epithelium and a small cytoplast (the cytoplasmic droplet) that remains attached to the sperm (Figure 1C). Although most residual bodies are likely engulfed and degraded by Sertoli cells (4749), a few putative residual bodies of unknown fate and unknown MGCA content are detected at stage IX (50). To determine whether these stage IX residual bodies participated in NS-MGCA egress, we investigated whether they contain antigenic MGCA and evade degradation by Sertoli cells. 
A

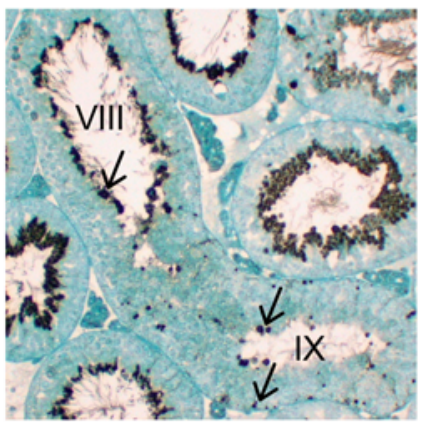

C

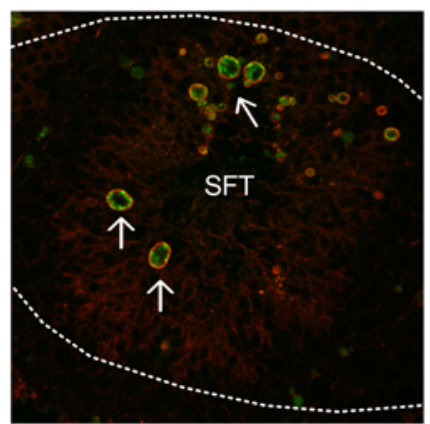

E

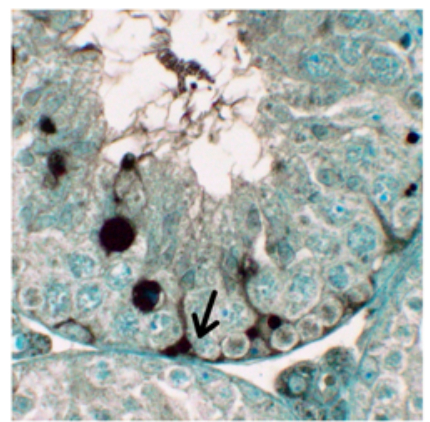

G

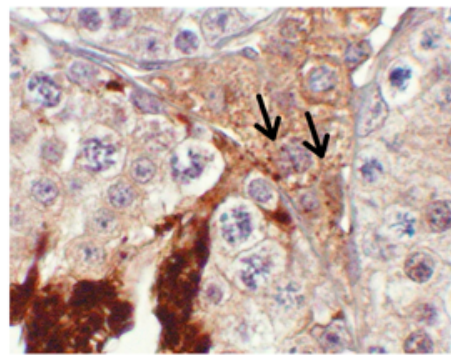

B $\stackrel{+}{\stackrel{+}{3}}$
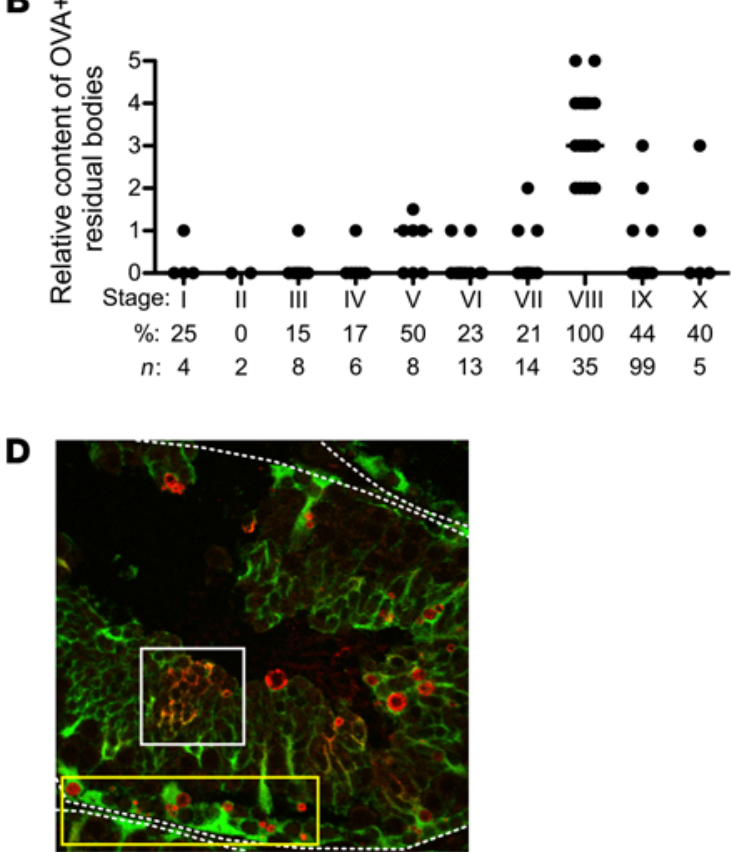

$\mathbf{F}$
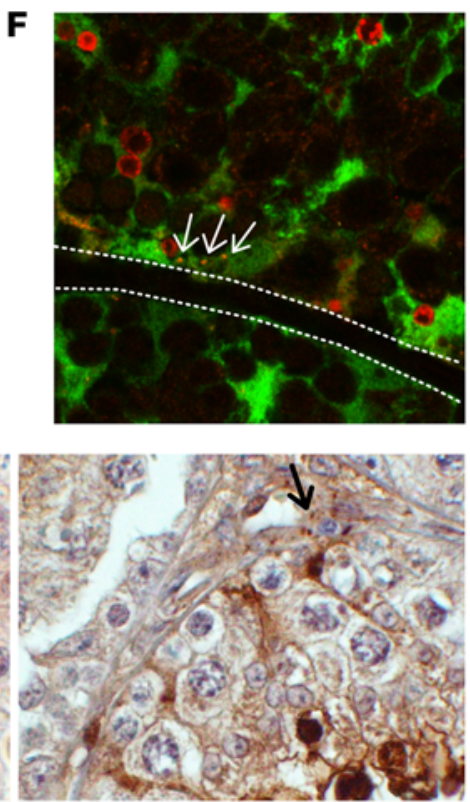

Figure 6. Stage-specific OVA localization and detection in residual bodies of OVAHi mice. (A) IP staining in a longitudinal seminiferous tubule section of an OVA-Hi mouse testis. Note: $\mathrm{OVA}^{+}$residual bodies (arrows) are with sperm at stage VIII, but are randomly distributed throughout the seminiferous tubule at stage IX. (B) OVA residual bodies with high OVA content are maximally detected at stage IX of the spermatogenic cycle (significance between stage IX and all other stages ranges from significant to highly significant). ${ }^{*} P<0.05$; ${ }^{* *} P<0.01 ;{ }^{* *} P<0.001$, Kruskal-Wallis test. (C) Residual bodies (indicated by arrows) in OVA-Hi mouse testis at stage IX: OVA (green) is enclosed by membrane-associated $t A C E$, a residual body marker (red). (D) Most OVA+ residual bodies (red) in OVA-Hi $\times$ Scx-GFP F1 testis at stage IX do not costain with Sertoli cell cytoplasm (green), including the very small residual bodies at the base of seminiferous tubules (yellow rectangle). Some OVA is inside the Sertoli cell cytoplasm (yellow) with plexiform distribution (white square). (E) Diffuse OVA IP staining confined to the basal cytoplasm of Sertoli cells in OVA-Hi mouse testis at stage IX (arrow). (F) OVA puncta (red) are inside the basal Sertoli cell cytoplasm (yellow, arrows) in OVA-Hi $\times$ ScxGFP F1 mouse testis at stage IX. (C) Small $\mathrm{OVA}^{+}$speckles detected by IP staining in the interstitial space of OVA-Hi mouse testes (arrows) at stage VIII (left) and stage IX (right). All images are representative of 3-4 experiments. Dotted white lines denote the boundary of seminiferous tubules. Original magnification, $\times 100(\mathbf{A})$; $\times 400$ (C-F); ×800 (G)
By immunoperoxidase (IP) and immunofluorescence (IF) microscopy, OVA was readily detected in the residual bodies in $20 \%$ of seminiferous tubule cross sections (39 of 194 seminiferous tubules in 3 experiments) of adult OVA-Hi mouse testes, consistent with the $18 \%$ of immune complexes detected in mice injected with Abs to OVA or LDH3 (Figure 2C). These were round and irregular structures of from less than 1 to 3 microns in diameter (Figure 6, A and C-F). By staging spermatogenesis based on the location and the rhomboid-shaped nuclei of the round spermatid, OVA-positive residual bodies were preferentially detected at stage IX $(n=35$, Figure $6 \mathrm{~B})$. In sagittal seminiferous tubule sections that span both stage VIII and stage IX (Figure 6A), OVA-positive residual bodies were attached to the apical sperm at stage VIII. After sperm release at stages VIII to
IX, numerous OVA-positive residual bodies of variable sizes were randomly distributed throughout the seminiferous epithelium at stage IX of the seminiferous cycle (Figure 6A).

The identity of the OVA-positive structures as residual bodies was further confirmed by their colocalization with the testicular isoform of angiotensin-converting enzyme (tACE), a membrane molecule expressed in the elongated spermatids (51). tACE formed a ring around OVA in the residual bodies (Figure 6C), indicating that the MGCA exist in the residual bodies at stage IX and that they are derived from both the cell membrane and the cytoplasm of elongated spermatids. In contrast, the residual bodies of OVA-Lo mice stained positively for tACE without detectable OVA (data not shown). To investigate how these residual bodies avoid degradation by Sertoli cells, we studied OVA-Hi $\times$ Scx-GFP 
A
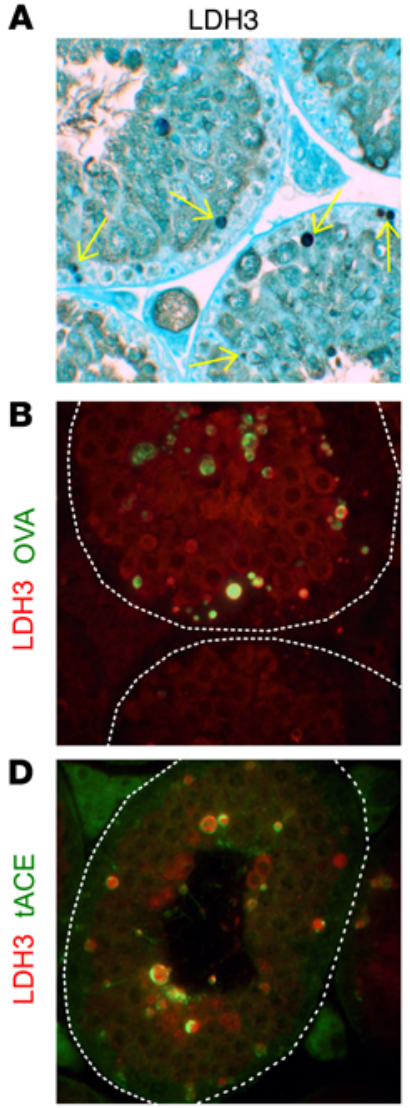
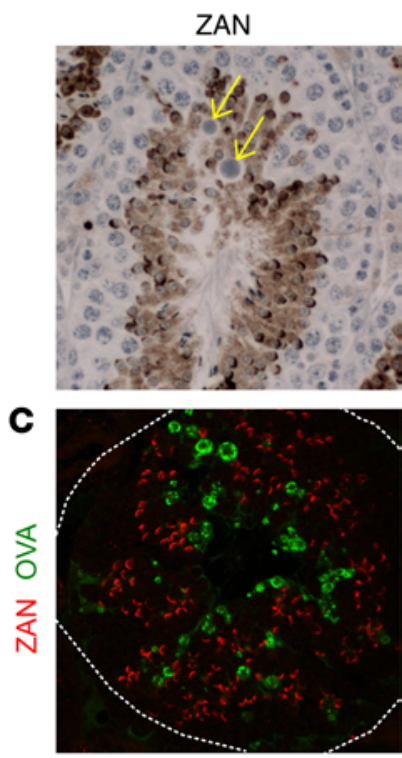

$\mathbf{E}$

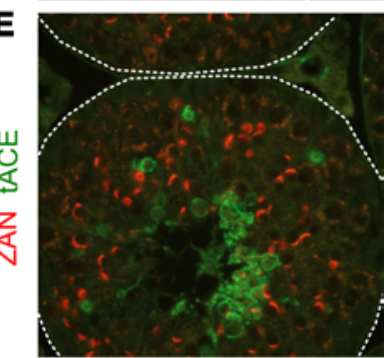

F1 mice that express GFP in the Sertoli cell cytoplasm under the Scx promoter (52). At stage IX, residual body internalization into the Sertoli cells was visualized as yellowish plexiform areas where the red OVA staining was colocalized with the Sertoli cell cytoplasmic GFP (Figure 6D). In contrast, numerous round and discrete OVA-positive residual bodies that did not colocalize with GFP were readily detected throughout the seminiferous tubules (Figure 6, D and F). Therefore, many residual bodies at stage IX were excluded from the Sertoli cell cytoplasm as they migrated to the basal seminiferous tubules (Figure 6, A and D).

Importantly, we also detected diffuse OVA staining confined to the basal cytoplasm of Sertoli cells in $12 \%$ of the seminiferous tubules at stage IX ( $n=286$ among 3 experiments), often surrounding the cell nuclei (Figure 6E), but occasionally appearing as a speckled pattern inside the $\mathrm{GFP}^{+}$Sertoli cell cytoplasm (Figure 6F). Moreover, in the interstitial spaces adjacent to the Sertoli cells that contain basal cytoplasmic OVA, we occasionally detected speckles of immunoreactive OVA (Figure 6G). Therefore, the OVA in the residual bodies appeared to enter the basal Sertoli cell cytoplasm before exiting the seminiferous tubules.

The detection of NS-MGCA in the residual bodies is not unique to the transgenic OVA antigen, as residual bodies contained LDH3 but not ZAN in WT BALB/c mouse testes (Figure 7A). This was confirmed by IF studies (Figure 7, B-E). In OVA$\mathrm{Hi}$ mouse testes, the $\mathrm{OVA}^{+}$residual bodies at stage IX also contained LDH3 (Figure 7B), but not ZAN (Figure 7C). In WT $\mathrm{BALB} / \mathrm{c}$ mice, with tACE as marker, LDH3 (Figure 7D) but not ZAN (Figure 7E) was detectable in the residual bodies.

Figure 7. Detection of LDH3 and ZAN in residual bodies of both OVA-Hi and BALB/C mouse testes. (A) LDH3 (left, arrows), but not ZAN (right, arrows), is detected in residual bodies of WT mouse testes at stage IX by IP staining (hematoxylin). In OVA-Hi mouse testes at stage IX, OVA (green) in residual bodies is colocalized with (B) LDH3 (red), but not with (C) ZAN (red). In WT testes, tACE (green) in the residual bodies is colocalized with (D) LDH3 (red), but not with (E) ZAN (red). All images are representative of 3-4 independent experiments. Dotted white lines denote the boundary of seminiferous tubules. Original magnification, $\times 400$.

In summary, our findings indicate that the residual bodies formed at stage IX of the spermatogenic cycle normally carry the tolerogenic NS-MGCA, but not the nontolerogenic S-MGCA. Instead of being totally degraded by the Sertoli cells, some intact residual bodies migrate basally in the seminiferous tubular epithelium and discharge their contents into the interstitial space via the basal cytoplasm of the Sertoli cells. Based on these immunohistochemical observations, along with the detection of NS-MGCA egress and tolerance status established in the above experiments, we conclude that NS-MGCA normally egress from the seminiferous tubules as cargo in the residual bodies at spermiation.

\section{Discussion}

We presented conclusive evidence that for some murine MGCA located behind the Sertoli cell barrier, systemic tolerance that requires FOXPp $3^{+}$Tregs is normally induced. This finding will add insight and clarity to our comprehension of the mechanism of testicular autoimmune disease pathogenesis and contribute to the understanding of human autoimmune infertility and vasectomy as well as immunity to CTA in male patients with cancer.

We discovered that MGCA can be either nonsequestered or sequestered in the seminiferous tubules, and systemic tolerance is only induced by the NS-MGCA that continuously egress from the seminiferous tubules with intact Sertoli cell barriers. The NS-MGCA are detected in the residual bodies that form at spermiation and egress into the testis interstitial space. We have also provided the first experimental evidence, to our knowledge, for the sequestration of other MGCA by showing that S-MGCA are neither detectable in the residual bodies nor tolerogenic. Although many of our experiments on MGCA egress relied on transgenic OVA as a surrogate MGCA, essentially all of the results were confirmed in WT mice; therefore, they are physiologically relevant.

The new paradigm of selective MGCA sequestration and tolerance (Figure 1D) is a clear departure from the current dogma of complete MGCA sequestration (Figure 1C); it indicates that the immune protection for MGCA in the testis depends on 2 processes: (a) systemic tolerance involving antigen-specific Tregs continuously maintained by the NS-MGCA in peripheral lymphoid organs, and (b) local mechanisms, including tissue barriers, for protecting the S-MGCA and limiting damage to germ cells during interstitial inflammation. The new paradigm, as well as the findings of Tregs as a critical peripheral tolerance mechanism, the immunological property of the residual bodies, and the identification of a new pathway of molecular transport from inside the seminiferous tubules into the testis interstitial space, will have a major impact on the current concepts of the testicular immune-privileged environment. 
We provided direct evidence for MGCA egress out of the seminiferous tubules by documenting the formation of immune complexes outside the Sertoli cell barrier following systemic LDH3 Ab transfer. Importantly, this occurred only in WT mice and did not occur in mice genetically deficient in LDH3. The fact that MGCA can react with circulating Abs also helps to clarify how EAO may be initiated despite the exclusion of the MGCA to the immune effectors in the presence of an intact Sertoli cell barrier. For example, in mice immunized s.c. with testis antigen in adjuvant, the NS-MGCA can form testicular immune complexes with circulating Abs produced in the immunogen-draining lymph nodes, and the MGCA can then be presented by testis antigen-presenting cells and reactivate the memory effector $\mathrm{T}$ cells. In cooperation with the effector cytokines and chemokines, the immune complexes could also direct the interstitial inflammation to the boundary of seminiferous tubules, where they disrupt the Sertoli cell barrier and invade the seminiferous tubules to perturb spermatogenesis. This unique sequence of EAO immunopathology has been observed in most experimental and human testicular autoimmune diseases $(32,53-56)$. The finding will also explain how Abs can enhance adoptive transfer of postvasectomy EAO (32) and participate in EAO progression (A.G.A. Paul and K.S.K. Tung, unpublished observations).

We also provided direct evidence for Tregs as the critical mechanism of physiological tolerance to NS-MGCA by documenting spontaneous EAO development in B6AF1-DEREG mice with transient Treg depletion. This conclusion was further supported by the preferential autoantibody response to LDH3 over ZAN in the Treg-depleted mice. Interestingly, when we investigated the sera from unilaterally vasectomized (uni-Vx) and antiCD25 mAb-treated mice that previously tested positively for ZAN autoantibodies (32), none of the sera IgG reacted with LDH3 (J. Harakal et al., unpublished observations). Collectively, our results strongly support the conclusion that Treg-dependent physiological tolerance is applicable to NS-MGCA and not to S-MGCA. It should also be noted that systemic tolerance was detectable for the NS-MGCA normally located in the elongated spermatids and the residual bodies. These cellular antigens have a late ontogeny of 5 weeks after birth. Thus, our findings also argue against another prevailing opinion, that systemic tolerance can only be initiated and maintained by MGCA present in the neonatal period (19). It should be noted that transiently Treg-depleted FOXP3-DTR mice or DEREG mice were also used to document a role for Tregs in tolerance for murine gastric autoantigens (57-59).

In addition to their relevance to testicular autoimmune diseases, the findings in this study may also affect tumor immunity (60). CTA expressed in many human tumors are currently considered highly immunogenic due to their sequestration and lack of systemic tolerance $(3,4)$. Contrary to this concept, we now show the CTA derived from different MGCA are not equally immunogenic. We posit that the differential tolerogenic properties of MGCA located behind the Sertoli cell barrier, predetermined by their sequestration and tolerance statuses in the normal testes, will likely influence the spontaneous or vaccine-induced immunity to CTA in tumors of male patients.

In autoimmune models induced by immunization with selfantigens in adjuvant, it is generally believed that the optimal immunogens are epitopes expressed at too low a level to main- tain tolerance, but sufficient to activate effector/memory T cells. Although this may apply to clinical autoimmune diseases triggered by molecular mimicry in the context of tissue inflammation (61), previous studies do not explain the mechanism of spontaneous EAO due to defective physiological tolerance normally maintained by the highly expressed and highly accessible tolerogenic NS-MGCA, as exemplified by EAO in the DEREG mice with transient Treg depletion. It also does not apply to the S-MGCA targeted in EAO after vasectomy (32). The selection of pathogenic autoantigens in a given autoimmune disease may therefore vary depending on the context of pathogenesis.

Although normal mice do not exhibit systemic tolerance to S-MGCA, a positive immune response may not occur or be detectable following S-MGCA exposure from their sequestered location. Indeed, the exposure of S-MGCA in uni-Vx mice did not result in a detectable response for many months (32). Instead, the sperm antigens that leaked from the inflamed epididymis after uni-Vx were found to elicit an efficient Treg response that rendered the mice specifically tolerant to MGCA $(32,36)$. Thus, simultaneous CD25 $\mathrm{Ab}$ treatment in the uni-Vx mice led to development of immune responses to MGCA and EAO, but not experimental autoimmune encephalomyelitis. The tolerance to S-MGCA occurred rapidly; thus, the uni-Vx mice developed severe EAO and $\mathrm{Ab}$ response to S-MGCA when Tregs were depleted by anti-CD25 mAbs at the same time as vasectomy, but not after 7 days (36). Because the EAO resistance state was reversed by neutralization with an anti-PD-L1 $\mathrm{Ab}$, but not an anti-PD-1 Ab (36), and because PD-L1, but not PD-1, is required for peripheral Treg induction $(62,63)$, we surmised that the post-Vx EAO resistance state was partially attributed to peripheral Treg induction against the exposed MGCA. Therefore, in addition to maintaining physiological tolerance to NS-MGCA, Tregs can be induced by the "foreign" S-MGCA in a manner similar to the Treg response to foreign antigens $(37,38)$.

By defining the exit pathway of NS-MGCA egress, we have also uncovered an unanticipated function for the residual bodies. Contrary to previous suggestions $(47,50,64)$, some residual bodies are excluded from the Sertoli cell cytoplasm and persist as they move to the basal region of the seminiferous epithelium, and they contain antigenic MGCA. Our study has not defined the precise cellular mechanisms by which the NS-MGCA are exported by the Sertoli cells, including the endocytic and microparticle pathways. Nonetheless, we have shown that residual body formation at stage IX is a physiological checkpoint that determines the sequestration and tolerogenicity between the 2 subsets of MGCA.

Although previous studies have shown that the residual bodies participate in transepithelial transport and molecular recycling in seminiferous tubular epithelium, the actual egress of the molecules out of the seminiferous tubules has not been documented. For example, residual bodies are involved in the transport of $\mathrm{Fe}^{++}$ and $\mathrm{Fe}^{++}$transport molecules released from mature germ cells at the apical seminiferous tubules, for utilization by the less mature meiotic germ cells located near the basal seminiferous tubule (65). In remodeling of the basal tight junctions, molecules have been shown to be recycled from the apical junctions of the Sertoli cell barrier in a process dependent on the endocytic uptake of the apical junction components into the ectoplasmic specialization of Sertoli cells $(45,66,67)$. In addition, because the residual bodies 
contain galectin-1 (68), a lectin that mediates activation-induced $\mathrm{T}$ cell apoptosis (69) and inhibits autoimmune arthritis and experimental autoimmune encephalomyelitis development $(70,71)$, they could also regulate local responses in the testis microenvironment, particularly near the location where premeiotic germ cells normally transit the Sertoli cell barrier.

The testis was established as an immune-privileged site by the seminal finding of prolonged allograft survival within the local testicular interstitial environment $(72,73)$. This was subsequently found to depend on Treg-dependent induction of systemic CD8 ${ }^{+} \mathrm{T}$ cell tolerance (73). We expect similar mechanisms to facilitate the induction and maintenance of physiological systemic tolerance to the NS-MGCA, involving interstitial somatic cells and cytokines that are also required for maintaining local immune privilege (25). In other words, similar local mechanisms will likely control both the afferent arm and the efferent arm of a testicular immune response. These include the immunoregulatory M2-like macrophages that dominate in the normal testis (25); the Sertoli cells, which can induce Treg conversion and expansion in a TGF- $\beta$ dependent manner (74-78); the Leydig cells, which produce testosterone and also induce Treg expansion $(79,80)$; and production of vitamin A, which also regulates other local responses $(81,82)$. This raises the question of how local tissue environments could influence systemic tolerance induction in the lymph nodes. Two recent studies provide a clue. First, diabetogenic antigens from the pancreatic islets are presented by antigen-presenting cells in numerous lymph nodes and are not confined to the pancreatic lymph node (83). Second, implantation of Sertoli cells by themselves under the kidney capsule was sufficient to prevent and ameliorate diabetes in NOD mice (77). Together, they raise the possibility that, in addition to providing a route for lymph node homing of soluble NS-MGCA molecules or tolerogenic antigen-presenting cells with their processed peptides to reach the regional lymph node, the testis afferent lymphatic vessels $(84,85)$ may allow immunoregulatory molecules produced in the "immune-privileged" interstitial environment to reach the regional lymph nodes, where they promote tolerogenic presentation of NS-MGCA and maintain antigen-specific Treg function.

In conclusion, the findings described herein of selective MGCA sequestration associated with the existence of NS-MGCA and the critical role for Tregs in maintaining systemic tolerance to NS-MGCA have shifted the existing paradigm and established new mechanisms responsible for immunological tolerance and autoimmunity to sperm- and testis-specific antigens. We predict that they will also have a potential impact on CTA responses. Our results should stimulate new strategies for future research on immune privilege, testicular physiology, male infertility, and vasectomy as well as cancer immunity and cancer vaccine design.

\section{Methods}

Mice, genotyping, fertility study. B6AF1 mice were from the National Cancer Institute. A/J, C57BL/6, SJL, and BALB/cByJ mice were from The Jackson Laboratory. DO11.10 mice were from Marcia McDuffie (University of Virginia). C57BL/6-DEREG mice from Katharina Lahl and Tim Sparwasser (Twincore) were crossed with $\mathrm{A} / \mathrm{J}$ mice to make B6AF1-DEREG mice. $L d h 3$ null mice were from Erwin Goldberg (41). Spermatid-specific OVA transgenic OVA-Hi and OVA-Lo mouse lines were made in our laboratory. C57BL/6 Scx-GFP mice expressing Sertoli cell cytoplasmic GFP under the scleraxis locus were from Ronen Schweitzer (Oregon Health Center, Portland, Oregon, USA) and were bred into OVA-Hi mice. DEREG mice and Scx-GFP mice were genotyped using tail DNA with published primers (41). OVA transgenic mice were genotyped using OVA primers (forward, 5 '-AGGCCTTGAGCAGCTTGAGAGTAT, and reverse, 5'-TGAGGAGATGCCAGACAGATT). All mice were bred in a specific pathogen-free facility and tested negative for fur mites, pinworm, and Helicobacter spp. Male fertility was determined by the number of pups borne by 2 females cohoused with each male mouse within 2 months.

Production of OVA transgenic mice and detection of OVA $m R N A$. To construct OVA transgenic mice, cDNA encoding full-length OVA protein was cloned into the BamHI site of the PPCEXV-1 protamine expression vector (a gift of Robert E. Braun, University of Washington, Seattle, Washington, USA) under the control of the mouse Prm1 $5^{\prime}$ promoter sequence. The transgenic mice were generated in the University of Virginia Genetically Engineered Murine Model Core. Two OVA transgenic lines were backcrossed 18 times to BALB/cByJ mice. Transgenic OVA mRNA was detected in total RNA extracted by the Perfect RNA Mini Total RNA Isolation Kit (Eppendorf) or the TRIzol Reagent (Life Technologies). Samples were treated with DNase I (Life Technologies), and mRNA sequences were amplified from total RNA (3-5 mg) by oligo-dT primed reverse transcription using the SuperScript Preamplification System (Life Technologies). The OVA sequence was determined by PCR.

Recombinant MGCA and MGCA Ab production. Mouse LDH3 was extracted from adult mouse testis homogenate by heating to $55^{\circ} \mathrm{C}$, followed by ammonium sulfate precipitation and DEAD-Sephadex chromatography. LDH3 crystals formed in $40 \%$ ammonium sulfate (86) were used to immunize rabbits. Serum LDH3 Ab IgG was isolated on the protein A column. Two ZAN antigens were prepared. Recombinant D3p18 (amino acids Cys ${ }^{4502}$ to Lys $^{4621}$ of the ZAN sequence; accession no. AAC26680 [GenPept database of GenBank at National Center for Biotechnology: https://www.ncbi.nlm.nih.gov/protein]) was expressed in E. coli (87), and rabbit Ab to D3p18 was generated by immunization with a recombinant glutathione $S$-transferase-D3p18 (GST-D3p18) fusion protein and purified by affinity chromatography to sequentially remove GST and isolate ZAN epitope-specific Abs (34). The second was the ZAN holoprotein purified from pig spermatozoa by affinity chromatography (88) using an Ab specific for the pig ZAN recombinant D3 domain (Ile ${ }^{1684}-$ Pro $^{1788}$; accession AAC48486 [GenPept database of GenBank at National Center for Biotechnology: https://www.ncbi.nlm.nih.gov/protein]). The ZAN holoprotein Ab isolated by protein $\mathrm{G}$ crossreacts with mouse and human ZAN (88). Antigen specificity of the ZAN Ab was confirmed by Western blot and by sperm acrosome binding on IF and immunoelectron microscopy $(34,88)$. IgG fraction of anti-OVA antiserum (MP Biochemicals) was isolated on a protein $\mathrm{G}$ column.

$A b$ injection. To detect MGCA egress from seminiferous tubules, mice were injected i.p. with rabbit Abs to mouse LDH3, ZAN D3p18 peptide, pig ZAN, OVA, or control rabbit IgG at $3.75 \mu \mathrm{g}$ IgG/g body weight (see Supplemental Table 1 for full list of reagents and catalog numbers). Nineteen hours later, mice were perfused with PBS. To localize rabbit IgG immune complexes with endogenous mouse immune complexes in DEREG mice at 8 weeks after DT treatment, mice were injected i.p. with rabbit anti-LDH3 Abs. Nineteen hours later, mouse IgG1 was colocalized with rabbit IgG on frozen testis sections. 
Immunization with testis homogenate in adjuvant. Testis homogenate was prepared and EAO was induced as previously described (24). Testis homogenate at $10 \mathrm{mg}$ dry weight in CFA $(4.5 \mathrm{mg} / \mathrm{ml}$ of $\mathrm{Myco}$ bacterium tuberculosis H37Ra, DIFCO) was injected in 1 hind footpad and 2 s.c. sites. Two $5 \mu \mathrm{g}$ i.p. injections of Bordetella pertussis extracts (provided by John Munoz, Rocky Mountain National Laboratories, Hamilton, Montana, USA) were administered i.p. on days 0 and +1 of immunization, and the mice were studied 3 weeks later.

Unilateral vasectomy and anti-CD25 mAb treatment. Uni-Vx was performed by occlusion and bisection of the left vas deferens. On days $-3,+3$, and +7 of surgery (day 0 ), mice were injected with $250 \mu \mathrm{g}$ of anti-CD25 mAb (clone PC61) (32). Serum Abs were determined 10 weeks later. Controls included sham uni-Vx mice injected with antiCD25 mAbs and uni-Vx mice injected with PBS.

DT treatment of B6AF1-DEREG mice. Tregs were depleted by i.p. injection of DT (lot 322326, Calbiochem) at $40 \mu \mathrm{g} / \mathrm{kg}$ body weight on days 0,2 , and 5 (59), and mice were studied from 1 to 8 weeks later. Controls included WT mice injected with DT and DEREG mice injected with PBS.

In vivo Treg reconstitution. $\mathrm{CD} 4^{+} \mathrm{CD} 25^{+}$Tregs were isolated from pooled lymph nodes and spleens of WT donors. Erythrocyte lysed spleen cells were enriched for the $\mathrm{T}$ cell fraction on a mouse $\mathrm{T}$ cell enrichment column ( $\mathrm{R} \& \mathrm{D}$ Systems). $\mathrm{CD} 4^{+} \mathrm{CD} 25^{-} \mathrm{T}$ cells were isolated using $\mathrm{CD}^{+} \mathrm{CD} 25^{+}$Microbeads (Miltenyi Biotec) and AutoMACS (Miltenyi Biotec). Treg-depleted B6AF1-DEREG mice were injected i.v. with $2 \times 10^{6}$ viable Tregs at the time of the first dose of DT. The purity of the isolated $\mathrm{CD} 4{ }^{+} \mathrm{CD} 25^{+}$cells was $97.5 \% \pm 2.2 \%$.

EAO histopathology scoring and spermatogenic cycle staging. After the testes were weighed, EAO severity was scored on Bouin's fixed and H\&E-stained paraffin-embedded sections as unknown samples by a pathologist (K.S.K. Tung). The score was determined by the summation of testicular and epididymal inflammation, percentage of abnormal spermatogenesis, and epididymal sperm loss (24). Inflammation was graded from 0 to $4(0=$ none, $1=$ very focal, and $4=$ extremely severe with necrosis). Scores 2 and 3 were increments between 1 and 4 . The epididymal sperm loss score was determined by the summation of the sperm loss scores in the vas deferens, caput, body, and cauda of the epididymis as normal sperm levels (score 0), partial loss (score 1), and complete loss (score 2) for each region of the epididymis. Staging of the spermatogenic cycle was performed on Bouin's fixed paraffin-embedded sections of OVA-Hi mouse testes stained with rabbit anti-OVA Abs by immunoperoxidase and counterstained with H\&E or periodic acidSchiff (PAS) with hematoxylin counter stain. 194 random tubule cross sections from 2 separate mice were photographed, and the images were staged based on criteria established in the literature (89).

Transgenic OVA antigen detection by ELISA. Testes were weighed, dissociated in RIPA buffer at $100 \mathrm{mg} / \mathrm{ml}(\mathrm{w} / \mathrm{v})$ in the presence of $1 \mathrm{mM}$ PMSF, $2 \mu \mathrm{g} / \mathrm{ml}$ aprotinin, $2 \mu \mathrm{g} / \mathrm{ml}$ leupeptin, and $37.5 \mu \mathrm{g} / \mathrm{ml}$ soybean trypsin inhibitor, and incubated for 1 hour at $4^{\circ} \mathrm{C}$. Tissue lysates were centrifuged at high speed for 20 minutes at $4^{\circ} \mathrm{C}$, and total protein concentration of the supernatants was determined by the BCA Protein Assay Kit (Pierce). To detect OVA, 96-well flat-bottom plates were coated with $4 \mu \mathrm{g} / \mathrm{ml}$ rabbit anti-OVA in phosphate buffer at $\mathrm{pH} 6.0$ overnight at $4^{\circ} \mathrm{C}$ and then blocked with $3 \%$ BSA-PBS. Serial dilutions of testis extract were incubated with the OVA Abs for 1 hour at room temperature, and the bound OVA Abs were detected by mouse antiOVA IgG1 $(4 \mu \mathrm{g} / \mathrm{ml})$, followed by biotinylated goat anti-mouse IgG1
(1:1000), SA-HRP (1:3000), and OPD substrates. The amount of OVA per testis weight was computed from an OVA standard curve.

LDH3 and ZAN Ab detection by ELISA. Recombinant LDH3 expressed by a mouse LDH3 clone inserted in expression vector pKK223-3 was grown in E. coli strain W3110 lacI ${ }^{q}$, inducted by $1 \mathrm{mM}$ IPTG, extracted by $600 \mathrm{mM} \mathrm{NaCl}, 10 \mathrm{mM}$ imidazole, $100 \mathrm{mM}$ Tris$\mathrm{HCl}, \mathrm{pH} 7.9,0.1 \mathrm{mg} / \mathrm{ml}$ of lysozyme (Roche Biochemicals), $2 \mathrm{mM}$ PMSF, and protease inhibitor cocktail (Roche Biochemicals), and isolated by AMP-agarose column as described (90). Autoantibodies to LDH3 or ZAN were detected on 96-well flat-bottom plates coated with recombinant LDH3 $(10 \mu \mathrm{g} / \mathrm{ml})$ or recombinant ZAN D3p18 $(10 \mu \mathrm{g} /$ $\mathrm{ml}$ ) in PBS for 18 to 24 hours at $4^{\circ} \mathrm{C}$. After the wells were washed and blocked with $1 \%$ BSA-PBS, experimental and control mouse sera were added to the wells at 1:100 and 1:300 dilutions for the LDH3 ELISA and 1:50 dilution for the ZAN ELISA in duplicate and incubated for 1 hour. After 6 washes, the wells were incubated with HRP-conjugated goat anti-mouse IgG (Southern Biotech) at 1:2,000 for 1 hour. After 8 washes, each well was reacted with OPD substrate solution (SigmaAldrich). The reaction was terminated with $2.5 \mathrm{~N} \mathrm{H}_{2} \mathrm{SO}_{4}$, and absorbance at $490 \mathrm{~nm}$ was determined with a microplate reader.

Testicular cell Ab detection by ELISA. Single cell suspensions from decapsulated adult testes were incubated with collagenase II (100 U/ $\mathrm{ml}$, Worthington Biochemical Corp.) and DNAase (10 $\mu \mathrm{g} / \mathrm{ml}$, SigmaAldrich) for 15 minutes at $34^{\circ} \mathrm{C}$ (91). $1 \times 10^{5}$ testicular cells were added to each of 96-well flat-bottom plates in $100 \mu \mathrm{l}$ of PBS, centrifuged for 5 minutes at $500 \mathrm{~g}$, fixed with fresh $2 \%$ paraformaldehyde for 1 hour, and blocked with $1 \%$ BSA-PBS twice for 1 hour each time. The air-dried and coated plates were stored at $-20^{\circ} \mathrm{C}$ for up to 4 months. After the plates were rehydrated with PBS containing 0.05\% Tween 20 (SigmaAldrich) and incubated with 1\% BSA-PBS for 1 hour, they were incubated with 1:100 and 1:300 dilutions of serum samples in duplicate for 1 hour. The plate-bound mouse IgG was detected as described above, and autoantibody concentrations were extrapolated from a standard curve of pooled mouse sera with high testicular cell autoantibody titers.

IF and IP studies. All Abs and their sources are listed in Supplemental Table 1 (supplemental material available online with this article; doi:10.1172/JCI89927DS1). Supplemental Table 2 contains the details of the IF and IP procedures for each figure. Described below are the different procedures used on paraffin-embedded or frozen tissue sections. Frozen sections were used exclusively for IF studies, and paraffinembedded sections were used for both IF and IP studies. For frozen sections, whole testes were snap-frozen in liquid nitrogen and 5- to 8- $\mu \mathrm{m}$ sections were prepared on a Leitz cryostat (Leica). For paraffin sections, the whole testes, fixed for 5 days in Bouin's fixative and embedded in paraffin, were deparaffinized and hydrated. Antigen retrieval was performed on $5-\mu \mathrm{m}$ paraffin sections by boiling in citrate buffer $\mathrm{(pH} 6)$ for 7 minutes in a microwave oven previously moistened with $\mathrm{H}_{2} \mathrm{O}$ and prewarmed. The slides were cooled and hydrated in $\mathrm{H}_{2} \mathrm{O}$ and then PBS.

For IF staining on frozen sections, the testis sections were fixed in ice-cold 1:1 acetone/ethanol, blocked with Tris- $\mathrm{NaCl}$ blocking buffer (PerkinElmer), 3\% $\mathrm{H}_{2} \mathrm{O}_{2}$ (EMD Chemicals), and $0.1 \% \mathrm{NaN}_{3}$ (ICN Biomedicals, Inc.), followed by a biotin-avidin blocking kit (Vector Laboratories), and incubated with $\mathrm{Ab}$ for 30 to 45 minutes at room temperature. For IF staining on paraffin sections, Ab incubation was performed at $4^{\circ} \mathrm{C}$ overnight. The biotinylated $\mathrm{Ab}$ staining was amplified by the TSA Biotin Tyramide Reagent Pack (PerkinElmer) and detected with neutralite avidin-Texas Red. 
For IP staining, endogenous peroxidase was first blocked with Peroxidase and Alkaline Phosphatase Blocking Reagent (Dako). Paraffinembedded tissue sections were incubated with $\mathrm{Abs}$ at $4^{\circ} \mathrm{C}$ overnight. After biotinylated $\mathrm{Ab}$ staining, the slides were incubated with AvidinBiotin Complex (Vector Laboratories). Peroxidase was detected by the 3,3'-diaminobenzidine tetrahydrochloride until optimal color staining and intensity were achieved. Counterstains were H\&E, PAS/hematoxylin, or methylene blue. After dehydration, the slides were mounted with Cytoseal (Richard-Allan Scientific). For OVA detection in OVA-Hi mouse testes, slides were exposed to $0.05 \%$ osmium vapor for 1 minute.

IF slides were examined and photographed on a Nikon Microphot-FXA fluorescence microscope and Nikon HB-10101AF mercury lamp and Olympus Q color 5 camera. Alternatively, they were examined with a Zeiss LSM 700 confocal microscope. The percentage of seminiferous tubules with positive immune complex staining was counted among 100 tubules per section. IP slides were examined with an Olympus BH2 light microscope and photographed with the Olympus Infinity 1 camera.

Biotin and FITC exclusion assays. Mice were injected with $10 \mu \mathrm{l}$ of $7.5 \mathrm{mg} / \mathrm{ml}$ of biotin (Thermo Scientific) in PBS containing $1 \mathrm{mM} \mathrm{CaCl}$ under the testis capsule, and 60 minutes later, the mice were perfused and the testes were snap-frozen. Testis sections were fixed in ice-cold 1:1 acetone/ethanol, stained with neutralite avidin-Texas Red. FITC exclusion was determined after i.v. injection of $100 \mu \mathrm{l}$ of $2 \mathrm{mg} / \mathrm{ml}$ of FITC (isomer I, $\mathrm{C}_{21} \mathrm{H}_{11} \mathrm{NO}_{5} \mathrm{~S}, M_{r} 389.39$, Anaspec Inc.) in PBS. Ninety minutes later, the testes were snap-frozen in liquid nitrogen, and $8-\mu \mathrm{m}$ testis sections were costained for FITC and occludin and studied by fluorescence microscopy. Normal testes served as negative controls, and testes with EAO induced by testis homogenate and adjuvant served as positive controls.

Sertoli cell barrier protein quantification. Frozen testes were homogenized in a lysing solution (Bio-Plex Lysis Kit, Bio-Rad), and the protein concentrations of the lysates were determined using the RC-DC Protein Assay (Bio-Rad) with BSA as a standard. Proteins expressed in the Sertoli cell tight junctions were quantified by immunoblotting performed as previously described (92). Approximately $35 \mu \mathrm{g}$ of protein extracted from frozen testes was used per lane for electrophoresis and immunoblotting. Immunoblot analysis was performed using a chemiluminescence kit prepared in-house (92), and images were acquired with a Fujifilm LAS4000 mini Luminescent Image Analyzer.
Transmission electron microscopy. Briefly, deeply anesthetized mice were perfused intracardially with saline, followed by $1.5 \%$ glutaldehyde in saline, as previously described (93). The testes were trimmed, further fixed by immersion in $3 \%$ glutaldehyde in cacodylate buffer, and embedded in epon. Thin sections were stained with uranyl acetate and lead citrate and examined with a Zeiss electron microscope.

Analysis of testicular macrophages by flow cytometry. Testicular cells were isolated from testes of DEREG mice injected with DT 8 weeks earlier. Testis cell suspensions were obtained as described above, and macrophages, gated on live, $\mathrm{CD} 45^{+}$and $\mathrm{CD} 11 \mathrm{~b}^{+}$cells were stained for M1-like macrophages by iNOS and MHC class II, and M2-like macrophages by F4/80, CD206, and IL-4R $\alpha$.

Statistics. Statistical differences between mouse groups were determined by the nonparametric Mann-Whitney $U$ test or the Kruskal-Wallis test with Dunn's post-tests using GraphPad Prism, version 5.0. The 1-tailed Fisher's exact test was used to assess differences in disease incidences. $P<0.05$ was considered statistically significant. Data represent mean \pm SEM.

Study approval. All animal studies were approved by the Animal Care and Use Review Board at the University of Virginia that follows the NIH Guidelines.

\section{Author contributions}

KSKT and JH designed the experiments. KSKT, JH, HQ, CR, JCHL, AGAP, KW, PP, CMG, RDS, EWPW, and PPR conducted the experiments and analyzed the data. KSKT, JH, USD, DMH, HT, EG, CYC, and WS provided experimental tools or generated the mouse models. KSKT and JH wrote the manuscript.

\section{Acknowledgments}

The study was supported by NIH RO1 AI 41236. The authors are grateful to Ka-Wai Mok and Weiliang Xia for their contributions. They are extremely appreciative of the very helpful advice from Rex Hess and Esther Meyron-Holtz.

Address correspondence to: Kenneth S.K. Tung, Department of Pathology, Carter Immunology Center, University of Virginia, Box 801386, Charlottesville, Virginia 22908, USA. Phone: 434.924.9194; E-mail:kst7k@virginia.edu.
1. Turek PJ, Lipshultz LI. Immunologic infertility. Urol Clin North Am. 1994;21(3):447-468.

2. Duan YG, et al. Immunodeviation towards a Th17 immune response associated with testicular damage in azoospermic men. Int J Androl. 2011;34(6 Pt 2):e536-e545.

3. Simpson AJ, Caballero OL, Jungbluth A, Chen YT, Old LJ. Cancer/testis antigens, gametogenesis and cancer. Nat Rev Cancer. 2005;5(8):615-625.

4. Whitehurst AW. Cause and consequence of cancer/testis antigen activation in cancer. Annu Rev Pharmacol Toxicol. 2014;54:251-272.

5. Xing Y, Hogquist KA. T-cell tolerance: central and peripheral. Cold Spring Harb Perspect Biol. 2012;4(6):doi:10.1101/cshperspect.a006957.

6. Anderson MS, Venanzi ES, Chen Z, Berzins SP, Benoist C, Mathis D. The cellular mechanism of Aire control of T cell tolerance. Immunity. 2005;23(2):227-239.
7. Garza KM, Agersborg SS, Baker E, Tung KS. Persistence of physiological self antigen is required for the regulation of self tolerance. J Immunol. 2000;164(8):3982-3989.

8. Setiady YY, et al. Physiologic self antigens rapidly capacitate autoimmune disease-specific polyclonal CD4+CD25+ regulatory T cells. Blood. 2006;107(3):1056-1062.

9. Vahl JC, et al. Continuous $\mathrm{T}$ cell receptor signals maintain a functional regulatory $\mathrm{T}$ cell pool. Immunity. 2014;41(5):722-736.

10. Tung KS. Mechanism of self-tolerance and events leading to autoimmune disease and autoantibody response. Clin Immunol Immunopathol. 1994;73(3):275-282.

11. Sakaguchi S, Yamaguchi T, Nomura T, Ono M. Regulatory T cells and immune tolerance. Cell. 2008;133(5):775-787.

12. Kim JM, Rasmussen JP, Rudensky AY. Regula- tory T cells prevent catastrophic autoimmunity throughout the lifespan of mice. Nat Immunol. 2007;8(2):191-197.

13. Garza KM, Agersborg SS, Baker E, Tung KS. Persistence of physiological self antigen is required for the regulation of self tolerance. JImmunol. 2000;164(8):3982-3989.

14. Lathrop SK, Santacruz NA, Pham D, Luo J, Hsieh CS. Antigen-specific peripheral shaping of the natural regulatory $\mathrm{T}$ cell population. JExp Med. 2008;205(13):3105-3117.

15. Samy ET, Wheeler KM, Roper RJ, Teuscher C, Tung KS. Cutting edge: autoimmune disease in day 3 thymectomized mice is actively controlled by endogenous disease-specific regulatory $\mathrm{T}$ cells. J Immunol. 2008;180(7):4366-4370.

16. Wheeler KM, Samy ET, Tung KS. Cutting edge: normal regional lymph node enrichment of antigen-specific regulatory $\mathrm{T}$ cells with autoim- 
mune disease-suppressive capacity. J Immunol. 2009;183(12):7635-7638.

17. Dym M, Fawcett DW. The blood-testis barrier in the rat and the physiological compartmentation of the seminiferous epithelium. Biol Reprod. 1970;3(3):308-326.

18. Hintz M, Goldberg E. Immunohistochemical localization of LDH-x during spermatogenesis in mouse testes. Dev Biol. 1977;57(2):375-384.

19. Mital P, Hinton BT, Dufour JM. The blood-testis and blood-epididymis barriers are more than just their tight junctions. Biol Reprod. 2011;84(5):851-858.

20. Russell LD, Ettlin RA, Sinha Hikim AP, Clegg ED. Histological and Histopathological Evaluation of the Testis. Int J Androl.1990;16(1):83.

21. Oakberg EF. Duration of spermatogenesis in the mouse and timing of stages of the cycle of the seminiferous epithelium. Am JAnat. 1956;99(3):507-516.

22. Johnson MH. Changes in the blood-testis barrier of the guinea-pig in relation to histological damage following iso-immunization with testis. JReprod Fertil. 1970;22(1):119-127.

23. Tung KS, Unanue ER, Dixon FJ. Pathogenesis of experimental allergic orchitis. II. The role of antibody. J Immunol. 1971;106(6):1463-1472.

24. Tung KS, Yule TD, Mahi-Brown CA, Listrom MB. Distribution of histopathology and Ia positive cells in actively induced and passively transferred experimental autoimmune orchitis. JImmunol. 1987;138(3):752-759.

25. Hedger MP. Macrophages and the immune responsiveness of the testis. J Reprod Immunol. 2002;57(1-2):19-34.

26. Fijak M, Meinhardt A. The testis in immune privilege. Immunol Rev. 2006;213:66-81.

27. Li N, Wang T, Han D. Structural, cellular and molecular aspects of immune privilege in the testis. Front Immunol. 2012;3:152.

28. Perheentupa J. APS-I/APECED: the clinical disease and therapy. Endocrinol Metab Clin North Am. 2002;31(2):295-320, vi.

29. Kekäläinen E, et al. A defect of regulatory $\mathrm{T}$ cells in patients with autoimmune polyendocrinopathy-candidiasis-ectodermal dystrophy. J Immunol. 2007;178(2):1208-1215.

30. Adams CE, Wald M. Risks and complications of vasectomy. Urol Clin North Am. 2009;36(3):331-336.

31. Köhler TS, Fazili AA, Brannigan RE. Putative health risks associated with vasectomy. Urol Clin North Am. 2009;36(3):337-345.

32. Wheeler K, et al. Regulatory T cells control tolerogenic versus autoimmune response to sperm in vasectomy. Proc Natl Acad Sci U S A. 2011;108(18):7511-7516.

33. Hardy DM, Garbers DL. Species-specific binding of sperm proteins to the extracellular matrix (zona pellucida) of the egg.J Biol Chem. 1994;269(29):19000-19004.

34. Tardif S, et al. Zonadhesin is essential for species specificity of sperm adhesion to the egg zona pellucida. J Biol Chem. 2010;285(32):24863-24870.

35 . Tung KS. Human sperm antigens and antisperm antibodies I. Studies on vasectomy patients. Clin Exp Immunol. 1975;20(1):93-104.

36. Rival C, et al. Regulatory T cells and vasectomy. JReprod Immunol. 2013;100(1):66-75.
37. Kim KS, et al. Dietary antigens limit mucosal immunity by inducing regulatory $T$ cells in the small intestine. Science. 2016;351(6275):858-863.

38. Betts RJ, et al. Influenza A virus infection results in a robust, antigen-responsive, and widely disseminated Foxp3+ regulatory $\mathrm{T}$ cell response. J Virol. 2012;86(5):2817-2825.

39. Tang H, Goldberg E. Homo sapiens lactate dehydrogenase $\mathrm{c}$ (Ldhc) gene expression in cancer cells is regulated by transcription factor $\mathrm{Sp} 1$, CREB, and CpG island methylation. J Androl. 2009;30(2):157-167.

40. Dutta RC, Goldberg E. Testis specific lactate dehydrogenase as target for immunoliposomes. Am J Reprod Immunol. 2008;60(1):26-32.

41. Odet F, et al. Expression of the gene for mouse lactate dehydrogenase $\mathrm{C}(\mathrm{Ldhc}$ ) is required for male fertility. Biol Reprod. 2008;79(1):26-34.

42. Lahl K, et al. Selective depletion of Foxp3+ regulatory T cells induces a scurfy-like disease. J Exp Med. 2007;204(1):57-63.

43. Hedger MP. Macrophages and the immune responsiveness of the testis. J Reprod Immunol. 2002;57(1-2):19-34

44. Rival C, et al. Functional and phenotypic characteristics of testicular macrophages in experimental autoimmune orchitis. J Pathol. 2008;215(2):108-117.

45. Yan HH, Mruk DD, Lee WM, Cheng CY. Blood-testis barrier dynamics are regulated by testosterone and cytokines via their differential effects on the kinetics of protein endocytosis and recycling in Sertoli cells. FASEB J. 2008;22(6):1945-1959.

46. Peschon JJ, Behringer RR, Brinster RL, Palmiter RD. Spermatid-specific expression of protamine 1 in transgenic mice. Proc Natl Acad Sci USA. 1987;84(15):5316-5319.

47. Smith BV, Lacy D. Residual bodies of seminiferous tubules of the rat. Nature. 1959;184:249-251.

48. Blanco-Rodríguez J, Martínez-García C. Apoptosis is physiologically restricted to a specialized cytoplasmic compartment in rat spermatids. Biol Reprod. 1999;61(6):1541-1547.

49. Nakanishi Y, Shiratsuchi A. Phagocytic removal of apoptotic spermatogenic cells by Sertoli cells: mechanisms and consequences. Biol Pharm Bull. 2004;27(1):13-16.

50. Morales CR, Hay N, El-Alfy M, Zhao Q. Distribution of mouse sulfated glycoprotein-1 (prosaposin) in the testis and other tissues. JAndrol. 1998;19(2):156-164.

51. Atanassova N, Lakova E, Bratchkova Y, Krasteva G, Donchev M. Expression of testicular angiotensin-converting enzyme in adult spontaneously hypertensive rats. Folia Histochem Cytobiol. 2009;47(1):117-122.

52. Muir T, et al. Integration of CREB and bHLH transcriptional signaling pathways through direct heterodimerization of the proteins: role in muscle and testis development. Mol Reprod Dev. 2008;75(11):1637-1652.

53. Bigazzi PE, Kosuda LL, Hsu KC, Andres GA. Immune complex orchitis in vasectomized rabbits. JExp Med. 1976;143(2):382-404.

54. Tung KS, Woodroffe AJ. Immunopathology of experimental allergic orchitis in the rabbit. JImmunol. 1978;120(1):320-328.
55. Tung KS, et al. The black mink (Mustela vison). A natural model of immunologic male infertility. JExp Med.1981;154(4):1016-1032.

56. Lehmann D, Temminck B, Da Rugna D, Leibundgut B, Sulmoni A, Müller H. Role of immunological factors in male infertility. Immunohistochemical and serological evidence. Lab Invest. 1987;57(1):21-28.

57. Zheng Y, et al. Regulatory T-cell suppressor program co-opts transcription factor IRF4 to control T(H)2 responses. Nature. 2009;458(7236):351-356.

58. Nyström SN, Bourges D, Garry S, Ross EM, van Driel IR, Gleeson PA. Transient Treg-cell depletion in adult mice results in persistent selfreactive $\mathrm{CD} 4(+) \mathrm{T}$-cell responses. Eur J Immunol. 2014;44(12):3621-3631.

59. Harakal J, Rival C, Qiao H, Tung KS. Regulatory T Cells Control Th2-Dominant Murine Autoimmune Gastritis. JImmunol. 2016;197(1):27-41.

60. Gjerstorff MF, Burns J, Ditzel HJ. Cancer-germline antigen vaccines and epigenetic enhancers: future strategies for cancer treatment. Expert Opin Biol Ther. 2010;10(7):1061-1075.

61. Luo AM, Garza KM, Hunt D, Tung KS. Antigen mimicry in autoimmune disease sharing of amino acid residues critical for pathogenic $\mathrm{T}$ cell activation. JClin Invest. 1993;92(5):2117-2123.

62. Francisco LM, et al. PD-L1 regulates the development, maintenance, and function of induced regulatory T cells. J Exp Med. 2009;206(13):3015-3029.

63. Riella LV, Paterson AM, Sharpe AH, Chandraker A. Role of the PD-1 pathway in the immune response. Am J Transplant. 2012;12(10):2575-2587.

64. Breucker H, Schäfer E, Holstein AF. Morphogenesis and fate of the residual body in human spermiogenesis. Cell Tissue Res. 1985;240(2):303-309.

65. Leichtmann-Bardoogo Y, et al. Compartmentalization and regulation of iron metabolism proteins protect male germ cells from iron overload. Am J Physiol Endocrinol Metab. 2012;302(12):E1519-E1530.

66. Smith BE, Braun RE. Germ cell migration across Sertoli cell tight junctions. Science. 2012;338(6108):798-802.

67. Vogl AW, Young JS, Du M. New insights into roles of tubulobulbar complexes in sperm release and turnover of blood-testis barrier. Int Rev Cell Mol Biol. 2013;303:319-355.

68. Dettin L, Rubinstein N, Aoki A, Rabinovich GA, Maldonado CA. Regulated expression and ultrastructural localization of galectin-1, a proapoptotic beta-galactoside-binding lectin, during spermatogenesis in rat testis. Biol Reprod. 2003;68(1):51-59.

69. Rabinovich GA, Toscano MA. Turning 'sweet' on immunity: galectin-glycan interactions in immune tolerance and inflammation. Nat Rev Immunol. 2009;9(5):338-352.

70. Rabinovich GA, et al. Recombinant galectin-1 and its genetic delivery suppress collagen-induced arthritis via T cell apoptosis. JExp Med. 1999;190(3):385-398.

71. Mari ER, et al. Galectin- 1 is essential for the induction of MOG35-55 -based intravenous tolerance in experimental autoimmune encephalomyelitis. Eur JImmunol. 2016;46(7):1783-1796.

72. Head JR, Neaves WB, Billingham RE. Immune 


\section{RESEARCH ARTICLE}

privilege in the testis. I. Basic parameters of allograft survival. Transplantation. 1983;36(4):423-431.

73. Nasr IW, et al. Testicular immune privilege promotes transplantation tolerance by altering the balance between memory and regulatory $\mathrm{T}$ cells. JImmunol. 2005;174(10):6161-6168.

74. Selawry HP, Cameron DF. Sertoli cell-enriched fractions in successful islet cell transplantation. Cell Transplant. 1993;2(2):123-129.

75. Suarez-Pinzon W, Korbutt GS, Power R, Hooton J, Rajotte RV, Rabinovitch A. Testicular sertoli cells protect islet beta-cells from autoimmune destruction in NOD mice by a transforming growth factor-beta1-dependent mechanism. Diabetes. 2000;49(11):1810-1818.

76. Dufour JM, Dass B, Halley KR, Korbutt GS, Dixon DE, Rajotte RV. Sertoli cell line lacks the immunoprotective properties associated with primary Sertoli cells. Cell Transplant. 2008;17(5):525-534.

77. Fallarino F, et al. Therapy of experimental type 1 diabetes by isolated Sertoli cell xenografts alone. JExp Med. 2009;206(11):2511-2526.

78. Campese AF, et al. Mouse Sertoli cells sustain de novo generation of regulatory $\mathrm{T}$ cells by triggering the notch pathway through soluble JAGGED1. Biol Reprod. 2014;90(3):53.

79. Fijak M, et al. Testosterone replacement effec- tively inhibits the development of experimental autoimmune orchitis in rats: evidence for a direct role of testosterone on regulatory $\mathrm{T}$ cell expansion. JImmunol. 2011;186(9):5162-5172.

80. Fijak M, et al. Influence of testosterone on inflammatory response in testicular cells and expression of transcription factor Foxp3 in $\mathrm{T}$ cells. Am J Reprod Immunol. 2015;74(1):12-25.

81. Hall JA, Grainger JR, Spencer SP, Belkaid Y. The role of retinoic acid in tolerance and immunity. Immunity. 2011;35(1):13-22.

82. Zhou R, Horai R, Mattapallil MJ, Caspi RR. A new look at immune privilege of the eye: dual role for the vision-related molecule retinoic acid. J Immunol. 2011;187(8):4170-4177.

83. Wan X, Thomas JW, Unanue ER. Class-switched anti-insulin antibodies originate from unconventional antigen presentation in multiple lymphoid sites. J Exp Med. 2016;213(6):967-978.

84. Hirai S, et al. The origin of lymphatic capillaries in murine testes. J Androl. 2012;33(4):745-751.

85. Svingen T, François M, Wilhelm D, Koopman P. Three-dimensional imaging of Prox1-EGFP transgenic mouse gonads reveals divergent modes of lymphangiogenesis in the testis and ovary. PLOS ONE. 2012;7(12):e52620.

86. Goldberg E. Amino acid composition and properties of crystalline lactate dehydrogenase $\mathrm{X}$ from mouse testes. J Biol Chem. 1972;247(7):2044-2048.

87. Tardif S, et al. Zonadhesin D3-polypeptides vary among species but are similar in Equus species capable of interbreeding. Biol Reprod. 2010;82(2):413-421.

88. Hickox JR, Bi M, Hardy DM. Heterogeneous processing and zona pellucida binding activity of pig zonadhesin. J Biol Chem. 2001;276(44):41502-41509.

89. Ahmed EA, de Rooij DG. Staging of mouse seminiferous tubule cross-sections. Methods Mol Biol. 2009;558:263-277.

90. LeVan KM, Goldberg E. Properties of human testis-specific lactate dehydrogenase expressed from Escherichia coli. Biochem J. 1991; 273( Pt 3):587-592.

91. Yule TD, Montoya GD, Russell LD, Williams TM, Tung KS. Autoantigenic germ cells exist outside the blood testis barrier. J Immunol. 1988;141(4):1161-1167.

92. Mruk DD, Cheng CY. Enhanced chemiluminescence (ECL) for routine immunoblotting: An inexpensive alternative to commercially available kits. Spermatogenesis. 2011;1(2):121-122.

93. Tung KS, Alexander NJ. Monocytic orchitis and aspermatogenesis in normal and vasectomized rhesus macaques (Macaca mulatta). Am J Pathol. 1980;101(1):17-29. 